OAK RIDGE

NATIONAL LABORATORY

MANAGED BY UT-BATTELLE

FOR THE DEPARTMENT OF ENERGY

\title{
Improved Processing of High Alloy Steels for Wear Components in Energy Generation Systems, Transportation and Manufacturing Systems
}

$9 / 30 / 2013$

Prepared by William H. Peter, Alan L. Liby, Wei Chen, Yukinori Yamamoto, Peter J. Blau 


\section{DOCUMENT AVAILABILITY}

Reports produced after January 1, 1996, are generally available free via the U.S. Department of Energy (DOE) Information Bridge.

Web site http://www.osti.gov/bridge

Reports produced before January 1, 1996, may be purchased by members of the public from the following source.

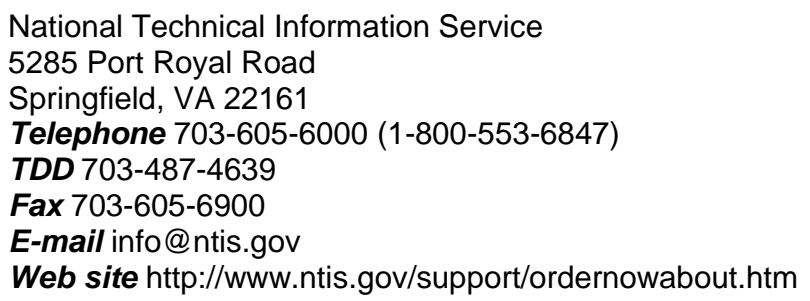

Reports are available to DOE employees, DOE contractors, Energy Technology Data Exchange (ETDE) representatives, and International Nuclear Information System (INIS) representatives from the following source.

Office of Scientific and Technical Information

P.O. Box 62

Oak Ridge, TN 37831

Telephone 865-576-8401

Fax 865-576-5728

E-mail reports@osti.gov

Web site http://www.osti.gov/contact.html

This report was prepared as an account of work sponsored by an agency of the United States Government. Neither the United States Government nor any agency thereof, nor any of their employees, makes any warranty, express or implied, or assumes any legal liability or responsibility for the accuracy, completeness, or usefulness of any information, apparatus, product, or process disclosed, or represents that its use would not infringe privately owned rights. Reference herein to any specific commercial product, process, or service by trade name, trademark, manufacturer, or otherwise, does not necessarily constitute or imply its endorsement, recommendation, or favoring by the United States Government or any agency thereof. The views and opinions of authors expressed herein do not necessarily state or reflect those of the United States Government or any agency thereof. 
Final Technical Report

\title{
Improved Processing of High Alloy Steels for Wear Components in Energy Generation Systems, Transportation and Manufacturing Systems
}

\author{
CPS Agreement No. 20978 \\ Project Period: July 2008 - May 2012 \\ Primary Investigators:
}

Drs. William H. Peter, Alan L. Liby, Wei Chen, Yukinori Yamamoto, Peter J. Blau

Oak Ridge National Laboratory

1 Bethel Valley Rd, Oak Ridge, Tennessee 37831

Industrial Participants:

Mr. Dave Peltier

Avure Technologies Inc.

Mr. Louis W. Lherbier and Mr. David J. Novotnak

Carpenter Technology Corporation

Date Published: September, 2013

\author{
Prepared by \\ OAK RIDGE NATIONAL LABORATORY \\ Oak Ridge, Tennessee 37831-6283 \\ managed by \\ UT-BATTELLE, LLC \\ for the \\ U.S. DEPARTMENT OF ENERGY \\ under contract DE-AC05-00OR22725
}




\section{Acknowledgments}

This report is based upon work supported by the Advanced Manufacturing Office of the U. S. Department of Energy under Award No.18716.

The authors would like to acknowledge Mr. David Harper for coordination of the tasks in the materials processing laboratory, Mr. Kevin Harper and Mr. Greg Cox for help with heat treatment, Mr. Jackie Mayotte for optical metallography and hardness measurements, Ms. Rita Ayers and Mr. Hiram Rogers for their help as administrative support including the formatting the report.

Research was also supported by ORNL's Shared Research Equipment (SHaRE) User Facility, which is sponsored by the Office of Basic Energy Sciences, U.S. Department of Energy. 


\section{CONTENTS}

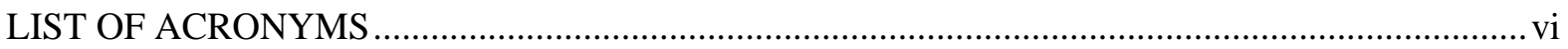

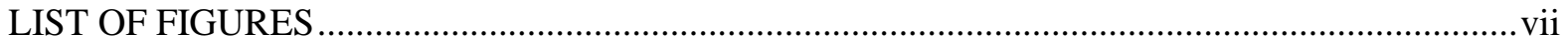

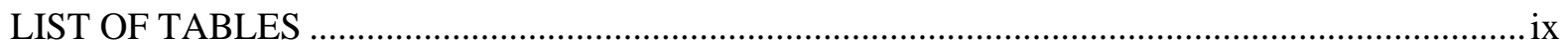

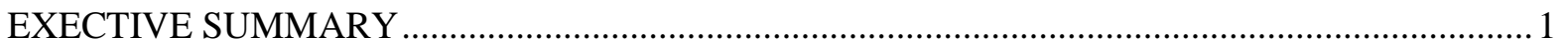

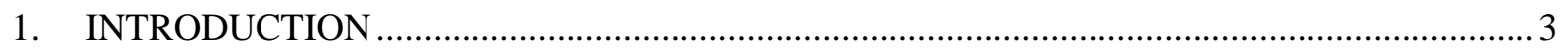

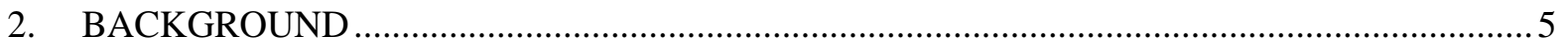

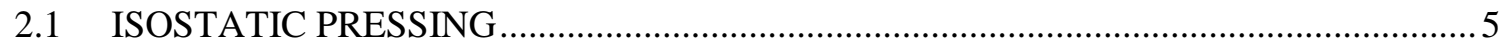

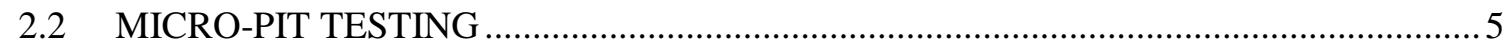

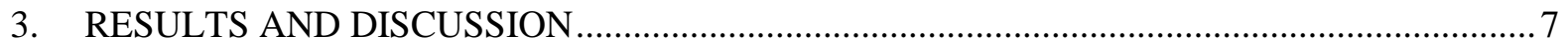

3.1 MATERIAL PROCUREMENTS AND SURVEY …............................................... 7

3.2 HEAT TREATMENT AND MIRCOSTRUCTURAL ANALYSIS ...............................

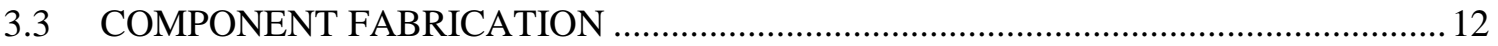

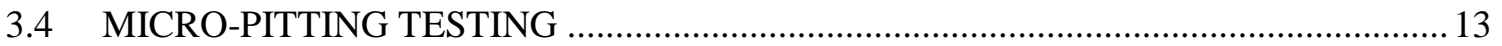

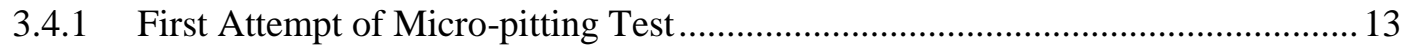

3.4.2 Optimized Test Conditions Dedicated Micro-pitting Formation........................... 20

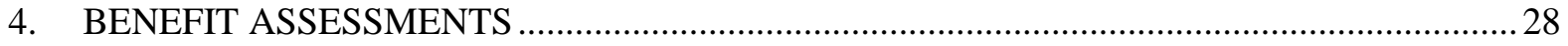

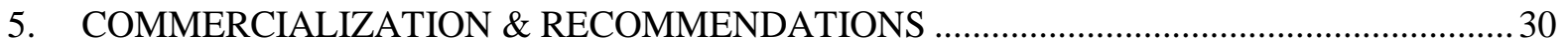

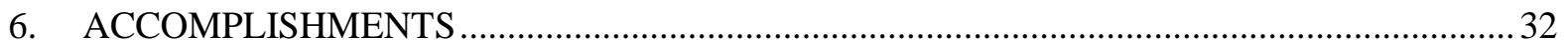

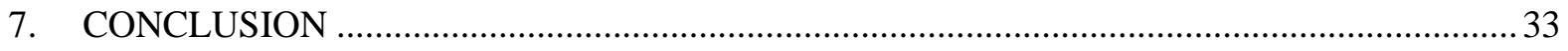

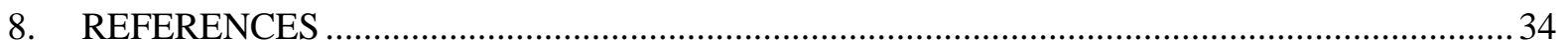




\section{LIST OF ACRONYMS}

$\begin{array}{ll}\text { AC } & \text { Air Cooled } \\ \text { AW } & \text { Air Warmed } \\ \text { BSE } & \text { Back-scattered Electron } \\ \text { CHIP } & \text { Cold isostatic press and sinter, followed by Hot Isostatic Press } \\ \text { CIP } & \text { Cold Isostatic Press } \\ \text { EDS } & \text { Energy Dispersive X-ray Spectroscopy } \\ \text { HIP } & \text { Hot Isostatic Press } \\ \text { MP } & \text { Micro-Pitting } \\ \text { MPR } & \text { Micro-Pitting test Rig } \\ \text { N } & \text { Newton } \\ \text { OQ } & \text { Oil Quenched } \\ \text { ORNL } & \text { Oak Ridge National Laboratory } \\ \text { P } & \text { Pitting } \\ \text { PM } & \text { Powder Metallurgy } \\ \text { SE } & \text { Secondary Electron } \\ \text { S } & \text { Sliding } \\ \text { SHaRE } & \text { Shared Research Equipment } \\ \text { SEM } & \text { Scanning Electron Microscopes } \\ \text { TBtu } & \text { Trillion British Thermal Units } \\ \text { UK } & \text { United Kingdom } \\ \text { XRD } & \text { X-Ray Diffraction }\end{array}$




\section{LIST OF FIGURES}

Figure

FIGURE 1. ILLUSTRATION OF THE MAJOR BEARING SYSTEMS IN A WIND TURBINE. .3

FIGURE 2. M. SMEETH OF PCS INSTRUMENTS PROVIDED A TUTORIAL TO FOUR ORNL STAFF MEMBERS AFTER INSTALLING THE MACHINE.

FIGURE 3. CLUSTER TO ROLLERS SURROUNDING A SMALLER CENTRAL TEST ROLLER (CHAMBER DOOR OPEN). THE TEMPERATURE PROBE ENTERS THE CHAMBER FROM THE UPPER LEFT.

FIGURE 4. CALCULATED PHASE EQUILIBRIUM OF THE ALLOYS (440C, 440XH, M62, AND 52100) BY JMATPRO. NOTE THAT THE CALCULATION RESULT OF M50 WAS SIMILAR TO THAT OF 52100, ALTHOUGH THE PREDICTED CARBIDES WERE NOT FE $\mathrm{C}_{3} \mathrm{BUT}_{23} \mathrm{C}_{6}(10 \%)+\mathrm{M}_{6} \mathrm{C}(4 \%)$ $+\mathrm{M}(\mathrm{C}, \mathrm{N})(2 \%)$ AT $600^{\circ} \mathrm{C}$

FIGURE 5. OPTICAL MICROGRAPHS OF THE AS-RECEIVED MATERIALS.........................................10

FIGURE 6. OPTICAL MACROGRAPHS OF THE HEAT-TREATED MATERIALS. ...................................10

FIGURE 7. SEM-BACK SCATTERED ELECTRON IMAGES OF THE HARDENED AND TEMPERED SPECIMENS

FIGURE 8. (A) DRAWING OF THE TEST SPECIMEN, AND (B) SPECIMENS AFTER ROUGH

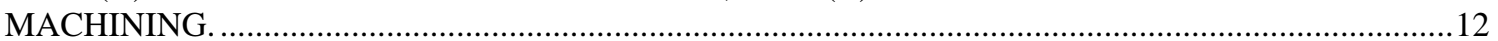

FIGURE 9. THE TEST SPECIMEN (440XH) AFTER FINAL MACHINING............................................13

FIGURE 10. MICROPIT PRODUCED OF A STEEL SURFACE DURING A PRELIMINARY EXPERIMENT.

FIGURE 11. (A) LOAD HISTORY, CORRESPONDING FRICTION COEFFICIENT, AND (B) WEAR RESULTS FOR M50.

FIGURE 12. AVERAGE FRICTION FOR EACH LOADING STEP FOR TWO TESTS OF EACH ALLOY.16

FIGURE 13. WEAR SUMMARY FOR TWO TESTS OF EACH ALLOY (AVERAGE WEAR FROM 2 RUNS). FOR ALL BUT THE 440XH WITH ITS HIGHER HARDNESS, THE RUNNING-IN WEAR DOMINATED STAGES 1 AND 2 AND WEAR IN STAGE 3 WAS LOWER.

FIGURE 14. WEAR DATA COMPARISON OF (A) THE TEST-INTERRUPTED SAMPLES FOR MICROSTRUCTURE CHARACTERIZATION AND (B) THE SAMPLES CONTINUED TO STAGE 4.

FIGURE 15. A PICTURE OF THE SAMPLE (440XH) BEFORE TESTING...................................................

FIGURE 16. SEM SECONDARY ELECTRON IMAGES OF THE SAMPLE SURFACE............................19

FIGURE 17. SEM BACK-SCATTERED ELECTRON IMAGES OF THE CROSS-SECTIONAL VIEW.......20

FIGURE 18. HIERARCHY OF SURFACE FEATURES OBSERVED ON METALLIC SURFACES SUBJECTED TO A COMBINATION OF ROLLING AND SLIDING ...............................................22

FIGURE 19. ABRASIVE WEAR GROOVES AND A FEW MICRO-PITS ON THE SURFACE OF AN M-50 STEEL ROLLER.

FIGURE 20. (A) A ROUNDED MICRO-PIT IN 440C STEEL, AND (B) DISTORTED MICRO-PITS IN M50 TOOL STEEL WHERE SHEAR HAS PARTLY OR FULLY CLOSED THEM, LEAVING FINE CRACKS AT BOTH ENDS 23

FIGURE 21. MICRO-PIT IN 52100 SHOWING (A) A BLOCKY NETWORK OF MICRO-CRACKS, AND (B), AN OBLIQUE VIEW OF MICRO-PITS IN THE SAME STEEL SPECIMEN SHOWING THE ARC-LIKE SHAPE OF A MICRO-CRACK THAT UNDERLIES A SHELF OF MATERIAL, AS SEEN AT THE RIGHT IN THE CROSS-SECTION IN FIGURE 7(A).

FIGURE 22. POLISHED AND ETCHED (2\% NITAL) CROSS-SECTION OF THE 52100 STEEL ROLLER SHOWING (A) A SERIES OF MICRO-FRACTURES EXTENDING FROM THE BOTTOM OF A MICRO-PIT LIKE THAT SHOWN IN FIGURE 21(A), AND (B) A ROW OF MICRO-CRACKS UNDERLYING THE SURFACE. THE OPEN END OF ONE SUCH CRACK CAN BE SEEN IN FIGURE 21(B). THE SLIP DIRECTION WAS RIGHT TO LEFT, SINCE THE PITS OPEN IN THE TENSILE FIELD FOLLOWING PASSAGE OF THE CONTACT PATCH.

FIGURE 23. SERIES OF MICRO-PITS ON 52100 STEEL THAT ARE CONNECTED BY MICROCRACKS. THE WIDTHS AND INTERIOR SCRATCHES WITHIN THE ABRASIVE GROOVES ARE 
SIMILAR IN SIZE TO THE BREATH OF THE MICRO-PITS SUGGESTING THAT WEAR DEBRIS FROM THE PITS CAN SERVE AS THIRD-BODY ABRADANTS. .....................................................24 FIGURE 24. PARTICLE OF 52100 STEEL ABOUT TO LEAVE THE SURFACE TO LEAVE A MICRO-

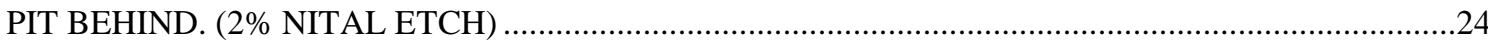

FIGURE 25. REDUCED FREQUENCY AND SIZE OF MICRO-PITS IN ALLOY 440XH. .......................25 FIGURE 26. HAIRLINE, TRANSVERSE CRACKS SUGGESTIVE OF MICRO-PITTING INITIATION IN THE RING SPECIMEN USED WITH M-50 ROLLERS. .26 FIGURE 27. WHITE LAYER ON A BEARING STEEL USED IN A WIND TURBINE GEARBOX BEARING SHOWING A SUBSURFACE VOID AND EXTENDING MICRO-CRACKS ASSOCIATED WITH THE NUCLEATION OF SPALLS.

FIGURE 28. MICRO-CRACKS AVOIDING CARBIDE PARTICLES AS THEY EXTEND FROM THE

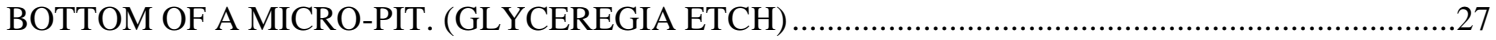

FIGURE 29. US WIND POWER MARKET GROWTH CURVE FROM 1998 TO 2011_.............................29

FIGURE 30. THE POWDER METALLURGY STRUCTURAL COMPONENTS MARKETS ........................30

FIGURE 31. PRODUCTION OF IRON AND STEEL FROM POWDER METALLURGY 1998-2010_...........31 


\section{LIST OF TABLES}

TABLE 1. COMPOSITIONS OF THE ALLOYS STUDIED (IN WEIGHT PERCENT, BALANCED FE) .......7 TABLE 2. PREDICTED PHASE EQUILIBRIUM AT $600^{\circ} \mathrm{C}$ (THE MATRIX IS $\alpha$-FE), TOGETHER WITH THE HARDNESS (ROCKWELL HARDNESS C) OF THE HEAT-TREATED SAMPLES......................

TABLE 3. HARDENING AND TEMPERING HEAT-TREATMENT …….....................................................

TABLE 4. HARDNESS OF THE INGOTS BEFORE AND AFTER HEAT-TREATMENT...............................

TABLE 5. HARDNESS OF THE SAMPLES BEFORE AND AFTER HEAT-TREATMENT …......................13

TABLE 6. MICRO-PITTING TEST PROTOCOL (FIRST TRIAL) …………….........................................15

TABLE 7. WEAR DATA OF THE ALLOYS (IN TERMS OF DISPLACEMENT, MM) FOR MICRO-

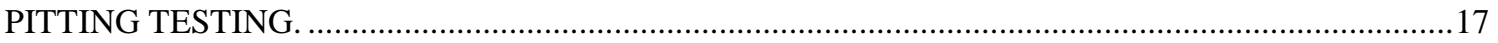

TABLE 8. MICRO-PITTING TEST PROTOCOL (SECOND TRIAL) ..........................................................2

TABLE 9. CATEGORIZATION OF FEATURES OBSERVED ON SURFACES OF STEEL TEST

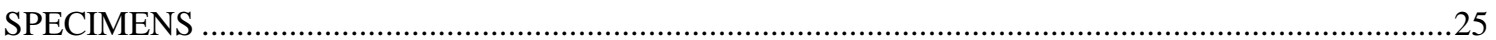

TABLE 10: INDUSTRIAL OPERATIONS WITH SIGNIFICANT ANNUAL WEAR CONSEQUENCES ....28 



\section{EXECTIVE SUMMARY}

Oak Ridge National Laboratory (ORNL), in Partnership with Avure Technologies Inc. and Carpenter Technology Corporation, explored methods to improve durability of steels considered essential to bearings in wind turbine applications by using hot isostatic pressing of steel alloy powders as a manufacturing method.

The objective of the project was to evaluate the wear mechanism of selected high alloy tool steels, 440C, 440XH, and M62, fabricated by powder metallurgy (PM) techniques via the hot isostatic press (HIP) process, and compare these results to two conventionally produced wrought alloys, 52100 and M50. Samples from the five candidate metals were characterized microstructurally via optical and scanning microscopy methods. A micro-pit wear testing machine was purchased and used to simulate bearing loads in a wind turbine gear box The candidate samples were subsequently heat treated in accordance with industrial protocols and machined into wear test specimens suitable for the micro-pit wear testing machine. . Wear testing produced surfaces that were subsequently analyzed for wear and damage using both optical microscopy and scanning electron microscopy methods.

Optimized rolling-sliding experiments with a relatively small slip ratio $(5 \%)$ and higher load $(650 \mathrm{~N})$ exhibited signs of abrasive wear on all specimens, but not all specimens had the same degree of micro-pitting damage. The conventional wrought 52100 steel had the most severe micro-pitting, consisting of distorted craters, many of which were connected by micro-cracks. Cross-sectional examination showed multiple micro-cracks extending into the alloy microstructure from the same pit. The depth of the cracks was several times the depth of the micro-pit. Type 440XH had the least amount of micro-pitting damage under similar test conditions. Micro-pits on 440HX were more localized and the micro-cracks associated with them tended to avoid carbides during propagation. Abrasive processes were observed on all the test specimens and were produced by debris from micopitting. The combination of micro-pitting in conjunction with abrasive processes could work together to worsen the surface damage, diminishing bearing service life.

The results suggest that there is a potential advantage of PM consolidated high alloy tool steels, such as $440 \mathrm{XH}$ produced via hot isostatic pressing to better resist micro-pitting when compared to other alloy types and wrought processing methods. 


\section{INTRODUCTION}

Friction and wear of components in transportation and energy generation equipment reduces system efficiencies, reduces component life, and parasitically consumes energy. Significant improvements in material wear translate to enduring energy savings and lower systems cost through lower frictional drag and reduced or predictable maintenance intervals. Oak Ridge National Laboratory, in Partnership with Avure Technologies Inc. and Carpenter Technology Corporation explored improvements in wear component performance through Hot Isostatic Pressing (HIP) of high alloy steel powders for wear applications specifically targeting wind turbine applications. Potential applications of the materials being studied in addition to wind turbine gear / bearing applications are listed below;

- Transportation: Hardened components are needed in any engine driven vehicle, whether the vehicle is a small car, a race car, a truck or an ocean vessel.

- General mechanical equipment: forging presses, steel rolling mills, machine tools; drivelines of mining equipment and heavy duty transmissions; earth moving equipment and heavy duty construction cranes. The combination of wear resistance and fatigue strength is a key characteristic of steels used for these applications.

There are multiple bearing systems in a wind turbine, such as the blade bearing, generator, gear box, main bearing, yaw bearing, and hydraulic system, as illustrated in Figure 1. Mechanical failures for wind turbines in the field are usually gear/bearing related, and a typical failure can cost $\$ 200,000$ to $\$ 400,000$. This cost drives a strong demand for advanced bearing steels with higher strength and wear-resistance.

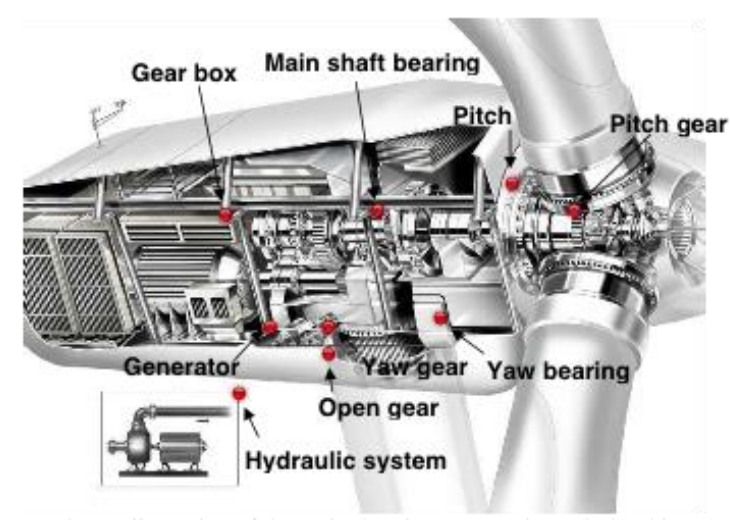

Figure 1. Illustration of the major bearing systems in a wind turbine.

High alloy steels have a potential to improve wear performance due to the precipitation of hard second-phases such as carbides or intermetallic compounds that are formed during solidification and heat treatment. However, it is difficult to control the size and distribution of the desirable, hard, second phases because the second phase manifestation is controlled by solidification dynamics and result in a course heterogeneous distribution when section thicknesses are large. Heterogeneous coarse carbide or intermetallic second phase distributions in the microstructure, due to segregation upon solidification, have a negative effect on wear resistance, reduce ductility, lower fracture toughness, and decreases fatigue life. 
The negative effect on wear is commonly attributable to the matrix microstructure surrounding the hard second phase having insufficient strength to retain the particle during high bearing load conditions. The surface area to volume ratio of the hard second phase particle also plays a role in matrix retention. The larger the second phase particle, the less surface area is available for the matrix to act upon it, hence large hard second phase particles are easier to pull from the matrix under high bearing load conditions, than small well distributed particles. Once the second phase is released from the metal matrix it becomes debris in the bearing contact zone, further acting upon the bearing surface as a wear particle.

Improvements in wear, with materials of the same composition, can be achieved by reducing the size and increasing the homogeneity of the distribution of the hard second phase. Powder metallurgy approaches remove the solidification constraints of the second phase distribution and allow the fabrication of large section thickness with an improved second phase size. Powders made via liquid metal processing retain the desired chemical composition due to rapid solidification during atomization. When the powders are consolidated into a large component with significant section thickness, the second phase particles remain small in size and homogeneous, resulting in improved wear.

In addition to the improved wear brought upon by a small homogeneous distribution of hard second phase particles, ductility, fracture toughness and fatigue performance are maintained at acceptable levels.

Alloy steel powders with a particle size of less than 5 micrometers were produced by Carpenter Technology Corporation using gas atomization. The powders were consolidated into test coupons using hot isostatic pressing.

The objective of this project was to test the wear resistance of selected high alloy tool steels, 440C, $440 \mathrm{XH}$, and M62, fabricated by consolidating powders via hot isostatic pressing (HIP) and compare those selected steels with commercial wrought tool steels, 52100 and M50, now used in wind turbine bearings. The evaluating test was a micro-pitting test, conducted using a contact fatigue testing machine that simulated wear in the bearing of a wind turbine gear box. The objective was to not only assess wear of the different materials in a relevant test, but to develop powder metallurgy fabrication methods that would allow commercialization of bearing manufacturing via HIP, if significant wear improvements were realized. 


\section{BACKGROUND}

\subsection{ISOSTATIC PRESSING}

Hot isostatic pressing, or HIP, is the process of reducing porosity of a casting or consolidating a powder metallurgy component by applying pressure at an elevated temperature over a moderate time interval. Pressure is applied with an inert gas in a pressure vessel, with pressures up to 2070 bar $(30,000 \mathrm{psi})$ and temperatures up $1320^{\circ} \mathrm{C}$ for steels and nickel based superalloys. In HIP processing, the gas pressure surrounds the work piece in all directions; isostatic pressure. Heat is applied usually through resistance heaters that are integral to the pressure vessel. If powder metallurgy components are to be successfully consolidated in the HIP process, the components must either be over $92 \%$ dense or must be encapsulated in a metal container (usually carbon steel) to act as a collapsible pressure boundary; otherwise the applied gas pressure permeates the partially consolidated powder negating the compression of the powder. The collapse of the can during the HIP cycle provides the physical pressure boundary, or sealing surface that consolidates the can's contents.

In many instances, the work piece may be cold isostatically pressed before HIPing to achieve a density greater than $55 \%$. Sintering will increase the density up to $92 \%+$. When hot isostatic pressing is preceded by cold isostatic pressing (CIP) and sintering the common acronym is CHIP. Powders are commonly measured/weighed and placed into polymer (polyurethane or rubber) bags before cold isostatic pressing, whereby the bag acts as the pressure boundary between the porous powder bed and the pressurizing media. CIP is performed at room temperature in a high pressure vessel. Fluid is used to fill the vessel and acts as the pressurizing medium.

Hot isostatic pressing has been performed on a wide range of materials systems with success. The process is commonly used in the production of aircraft components from powders, or for reducing porosity in cast components. Avure has demonstrated improvements of 30-50\% in wear performance of HIPed powder metallurgy components compared to conventionally processed steel.

\subsection{MICRO-PIT TESTING}

Evaluation of wear mechanisms for wind turbine gear boxes indicates that a new contact fatigue testing machine was required to investigate the durability of current and promising new experimental alloy steels in this project. Based upon an extensive survey of current commercial wear test systems, a dedicated micro-pitting test machine (MPR) produced by PCS Instruments was selected and purchased (Figure 2). The specialized bearing test apparatus was based on a design from Imperial College, London, and enables a highly-flexible, protocol-scripted approach to rolling contact fatigue testing, under lubricated environments such as those in wind turbine gearboxes, automotive power transmissions, and rolling bearings in general. It is especially designed to produce a form of localized surface damage called 'micro-pitting,' which is common in wind turbine gearboxes. The micro-pitting tester provided the ability to produce micro-pitting damage of the type observed on wind turbine gearbox bearings to the test matrix of steels.

The basic components of the machine are twin motors, a torque-monitored drive train, and a specimen chamber (Figure 3) that holds three outer rollers and in a smaller inner roller the test specimen of interest. Exclusive of the computer, the machine is approximately the size of a large refrigerator and weighs approximately $170 \mathrm{~kg}(375 \mathrm{lb})$. The maximum applied load to test specimens is $1250 \mathrm{~N}$ and the maximum surface speed between rollers is $4.0 \mathrm{~m} / \mathrm{s}$. The maximum lubricant temperature is 135 ${ }^{\circ} \mathrm{C}$. Conditions from pure slip to nearly pure rolling without slip are achievable by adjusting the relative rotational speeds of inner and outer rollers. Two software applications were included. One 
enabled the user to establish a script of operating instructions used to control each test segment. The second was the MPR test control software that ran whatever script that was selected.

Since conditions in a bearing can vary depending on the machine components around it and how it is used, the ability to adjust load, speed, temperature, and duration of testing is essential. In order to prepare the MPR to evaluate alloys developed in the project, conditions for measuring the effects of processing and alloy composition on resistance to micro-pitting were first established. For each experimental alloy specimen, a corresponding material and heat treatment of the outer rollers that apply the contact force to the test alloy were selected. The surface hardness of the outer rollers was higher than that of the experimental specimen for each case. Development efforts of processing protocols was also developed for each pairing of outer work rollers and alloy specimens, guided by typical wind turbine gearbox operating conditions based on industry information.

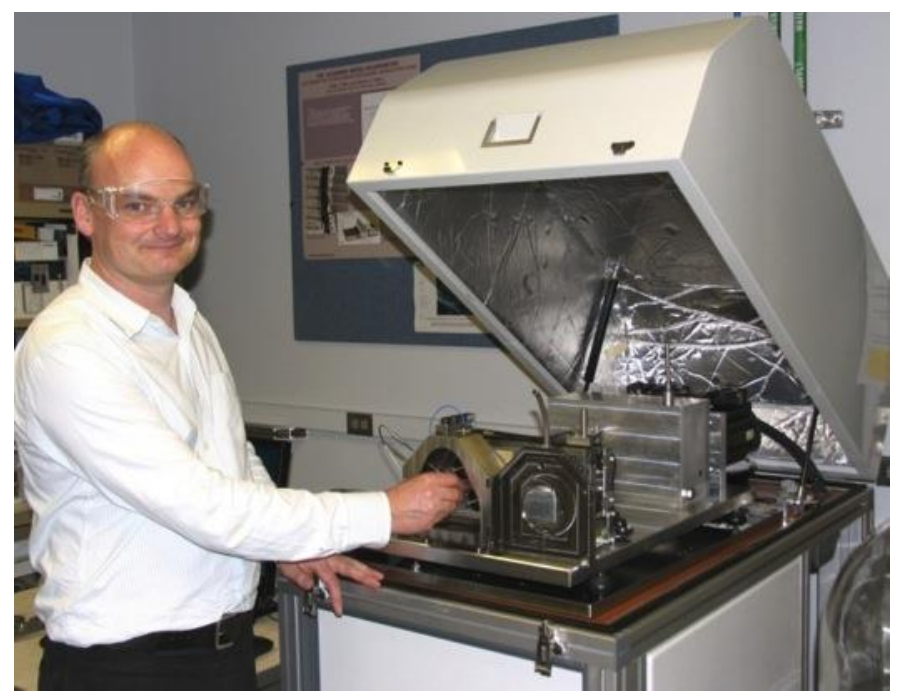

Figure 2. M. Smeeth of PCS Instruments provided a tutorial to four ORNL staff members after installing the machine.

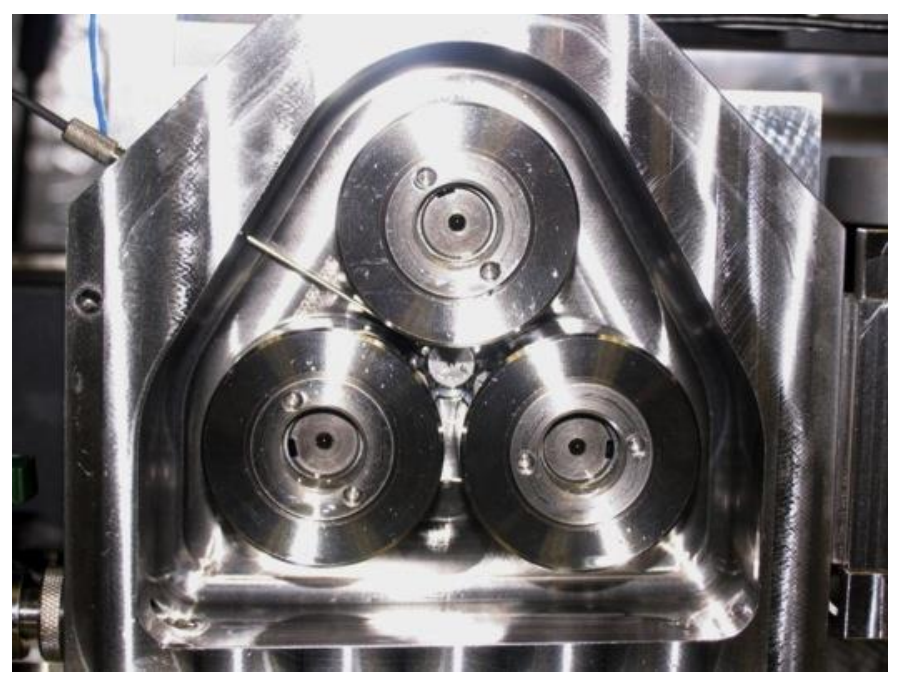

Figure 3. Cluster to rollers surrounding a smaller central test roller (chamber door open). The temperature probe enters the chamber from the upper left. 


\section{RESULTS AND DISCUSSION}

\subsection{MATERIAL PROCUREMENTS AND SURVEY}

Candidate bearing alloys to be examined in this project were selected and are shown in Table 1 below. The first 3 alloys; 440C, 440-XH and M62 are Carpenter Micro-Melt powder metallurgy alloys. The latter two alloys in the table are standard bearing alloys made by cast-and-wrought process. Type 52100 is a long-standing composition usually produced by air melting, but is also available as vacuum melted material. M-50 is usually vacuum-melted.

Table 1. Compositions of the alloys studied (in weight percent, balanced Fe)

\begin{tabular}{lcccccccc}
\hline Alloy & $\mathbf{C}$ & $\mathbf{M n}$ & $\mathbf{S i}$ & $\mathbf{C r}$ & $\mathbf{N i}$ & $\mathbf{M o}$ & $\mathbf{V}$ & $\mathbf{W}$ \\
$\mathbf{4 4 0 C}$ & $0.95 / 1.20$ & 1.00 & 1.00 & $16 / 18$ & - & 0.75 & - & - \\
$\mathbf{4 4 0 - X H}$ & 1.60 & 0.50 & 0.40 & 16 & 0.35 & 0.80 & 0.45 & - \\
M62 & 1.30 & 0.70 & 0.60 & 3.75 & - & 10.50 & 2.00 & 6.25 \\
$\mathbf{5 2 1 0 0}$ & $0.98 / 1.10$ & $0.25 / 0.45$ & $0.15 / 0.35$ & $1.30 / 1.60$ & - & - & - & - \\
M-50 & $0.77 / 0.85$ & 0.35 & 0.25 & $3.75 / 4.25$ & 0.10 & $4.00 / 4.50$ & $0.90 / 1.10$ & - \\
\hline
\end{tabular}

Theoretical phase equilibrium of the alloys calculated from JMatPro (a computational thermodynamic calculation tool) is shown in Figure 4. The equilibrated second-phase carbides at $600^{\circ} \mathrm{C}$ are quite different with each alloy: $18 \%$ of $\mathrm{M}_{23} \mathrm{C}_{6}$ in $440 \mathrm{C} ; 12 \%$ of $\mathrm{M}_{7} \mathrm{C}_{3}$ plus $8 \%$ of $\mathrm{M}_{23} \mathrm{C}_{6}$ in $440 \mathrm{XH} ; 22 \%$ of $\mathrm{M}_{6} \mathrm{C}$ plus $6 \%$ of $\mathrm{M}_{23} \mathrm{C}_{6}$ plus $4 \%$ of $\mathrm{M}(\mathrm{C}, \mathrm{N})$ in $\mathrm{M} 62 ; 14 \%$ of $\mathrm{Fe}_{3} \mathrm{C}$ in 52100 , and $10 \%$ of $\mathrm{M}_{23} \mathrm{C}_{6}$ plus $4 \%$ of $\mathrm{M}_{6} \mathrm{C}$ plus $2 \%$ of $\mathrm{M}(\mathrm{C}, \mathrm{N})$ in $\mathrm{M} 50$ (not in the figure). The $600^{\circ} \mathrm{C}$ temperature graphs, in the predictive model were used to mimic tempered conditions of bearing steels. The M62 alloy shows the largest total amount of second-phase carbides, and the 52100 the lowest, among the alloys studied. JMatPro does not predict the size and distribution of equilibrium phases, only the amount. These calculated results were consistent with the microstructure characterization and XRD analysis of the samples after hardening/ tempering heat-treatments. Calculations show that some fine carbides in 440C, 440XH and M62 formed by the rapid solidification of the gas atomization process do not completely dissolve at temperatures very close to the melting point of these alloys.

Table 2 summarizes the phase equilibrium of the alloys at $600^{\circ} \mathrm{C}$ predicted by JMatPro, together with the corresponding hardness of the heat-treated alloys. Note that the predicted amounts of the secondphases do not precisely reflect the actual amounts of the phases in the heat-treated samples, but could be used for comparison purposes. The comparison indicates that the hardness of the materials tends to increase with increasing the total amount of second-phases, except for M50. The M50 had a heat treatment that resulted in a higher carbon austenite than the other alloys of the test matrix. The martensitic transformation for M50 therefore resulted in a high carbon martensite and the hardness value is dominated by the matrix hardness.

Table 2. Predicted phase equilibrium at $600^{\circ} \mathrm{C}$ (the matrix is $\alpha-\mathrm{Fe}$ ), together with the hardness (Rockwell hardness $\mathrm{C}$ ) of the heat-treated samples.

\begin{tabular}{|c|c|c|c|c|c|c|c|}
\hline \multirow{2}{*}{ Alloy } & \multicolumn{6}{|c|}{ Second-phases, wt\% } & \multirow{2}{*}{ HRC } \\
\hline & $M_{23} C_{6}$ & $\mathbf{M}_{7} \mathbf{C}_{3}$ & $\mathbf{M}_{6} \mathbf{C}$ & $\mathbf{M}(\mathbf{C}, \mathbf{N})$ & $\mathrm{Fe}_{3} \mathrm{C}$ & Total & \\
\hline $440 \mathrm{C}$ & 18 & - & - & - & - & 18 & $64.0 \pm 0.3$ \\
\hline $440-\mathrm{XH}$ & 8 & 12 & - & - & - & 20 & $64.3 \pm 0.4$ \\
\hline M62 & 6 & - & 22 & 4 & - & 32 & $65.9 \pm 0.4$ \\
\hline 52100 & - & - & - & - & 14 & 14 & $60.0 \pm 0.4$ \\
\hline M-50 & 10 & - & 4 & 2 & - & 16 & $66.4 \pm 0.2$ \\
\hline
\end{tabular}




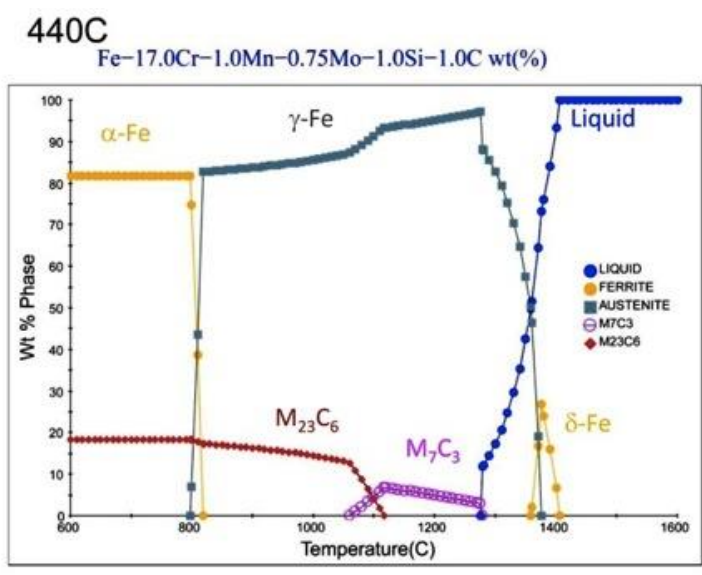

$440 \mathrm{XH}$

$\mathrm{Fe}-16.0 \mathrm{Cr}-0.5 \mathrm{Mn}-0.8 \mathrm{Mo}-0.35 \mathrm{Ni}-0.4 \mathrm{Si}-0.45 \mathrm{~V}-1.6 \mathrm{C}$ wt(\%)

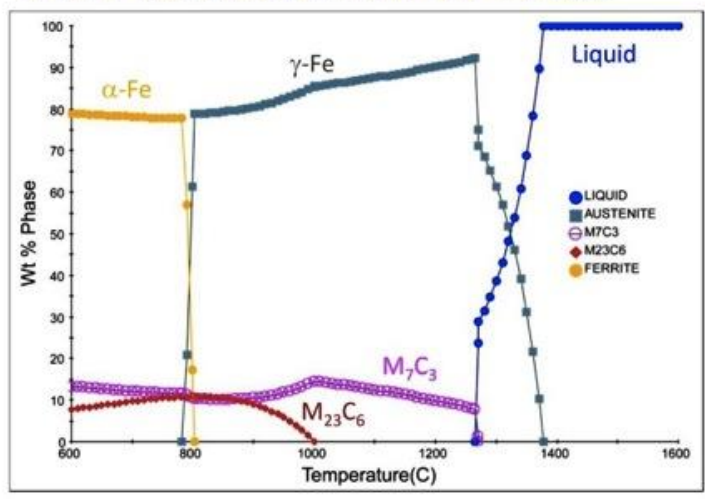

M62

$\mathrm{Fe}-3.75 \mathrm{Cr}-0.7 \mathrm{Mn}-10.5 \mathrm{Mo}-0.6 \mathrm{Si}-2.0 \mathrm{~V}-6.25 \mathrm{~W}-1.3 \mathrm{C}$ wt(\%)

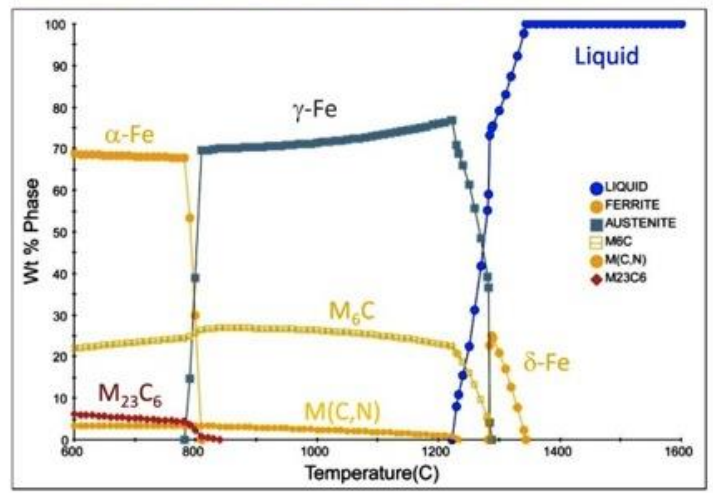

52100

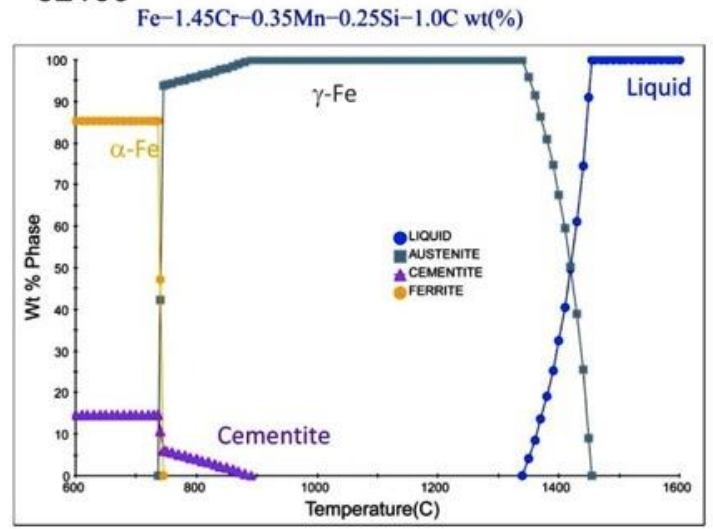

Figure 4. Calculated phase equilibrium of the alloys (440C, 440XH, M62, and 52100) by JMatPro.

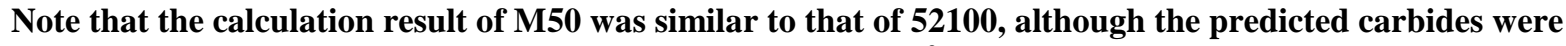
not $\mathrm{Fe}_{3} \mathrm{C}$ but $\mathrm{M}_{23} \mathrm{C}_{6}(10 \%)+\mathrm{M}_{6} \mathrm{C}(4 \%)+\mathrm{M}(\mathrm{C}, \mathrm{N})(2 \%)$ at $600^{\circ} \mathrm{C}$.

\subsection{HEAT TREATMENT AND MIRCOSTRUCTURAL ANALYSIS}

Three HIPed powder metallurgy (PM) ingots and a cast-and-wrought 52100 ingot arrived at ORNL in the normalized condition. The M-50 ingot was purchased from a commercial vendor also in the normalized condition. A small piece was sectioned from each alloy bar in the as-received ingot for microstructural characterization and hardness measurement. Additional pieces of each material were heat-treated to the hardened and tempered condition. After hardening and tempering, the samples were again measured for hardness and microstructurally characterized. The heat-treatments are summarized in Table 3.

Table 3. Hardening and tempering heat-treatment

\begin{tabular}{|c|c|c|}
\hline Alloy & Hardening & Tempering \\
\hline $\begin{array}{c}440 \mathrm{C} \text { and } \\
440 \mathrm{XH}\end{array}$ & $\begin{array}{l}1925 \mathrm{~F}(1052 \mathrm{C}) / 2 \mathrm{~h} / \mathrm{AC} \\
+-100 \mathrm{~F}(-73 \mathrm{C}) / 1 \mathrm{~h} / \mathrm{AW}\end{array}$ & $300 \mathrm{~F}(149 \mathrm{C}) / 1 \mathrm{~h} / \mathrm{AC}$ \\
\hline M62 & $\begin{array}{c}1525 \mathrm{~F}(829 \mathrm{C}) / 10 \mathrm{~min} \text { (preheat) } \\
\rightarrow 2150 \mathrm{~F}(1177 \mathrm{C}) / 5 \mathrm{~min} / \mathrm{AC}\end{array}$ & $\begin{array}{c}1200 \mathrm{~F}(649 \mathrm{C}) / 2 \mathrm{~h} / \mathrm{AC}(\text { stress relief }) \\
+1050 \mathrm{~F}(566 \mathrm{C}) / 2 \mathrm{~h} / \mathrm{AC}, \mathrm{x} 3\end{array}$ \\
\hline 52100 & $\begin{array}{c}1425 \mathrm{~F}(775 \mathrm{C}) / 4 \mathrm{~h} / \mathrm{AC} \\
+1540 \mathrm{~F}(838 \mathrm{C}) / 15 \mathrm{~min} / \mathrm{OQ} \\
\end{array}$ & $300 \mathrm{~F}(149 \mathrm{C}) / 1 \mathrm{~h} / \mathrm{AC}$ \\
\hline M50 & $\begin{array}{l}2025 \mathrm{~F}(1107 \mathrm{C}) / 5 \mathrm{~min} \rightarrow \text { quench in a } \\
\text { salt bath at } 1050-1150 \mathrm{~F}(566-621 \mathrm{C}) \\
\quad \rightarrow \text { AC to below } 150 \mathrm{~F}(66 \mathrm{C})\end{array}$ & $1000 \mathrm{~F}(538 \mathrm{C}) / 2 \mathrm{~h} / \mathrm{AC}, \mathrm{x} 3$ \\
\hline
\end{tabular}


Note that the micro-pitting specimens for this work were rough machined prior to heat-treatment. After the tempering process, the specimens were ground to meet the specimen geometry. The resulting hardness values of the ground specimens are summarized in Table 4.

Table 4. Hardness of the ingots before and after heat-treatment

\begin{tabular}{ccc}
\hline \multirow{2}{*}{ Alloy } & \multicolumn{2}{c}{ Hardness Rockwell C (Micro-Vickers hardness) } \\
\cline { 2 - 3 } & as received & heat-treated \\
\hline $440 \mathrm{C}$ & $40.6 \pm 0.9(399 \pm 9)$ & $67.0 \pm 0.9(905 \pm 31)$ \\
\hline $440 \mathrm{XH}$ & $41.0 \pm 1.0(403 \pm 10)$ & $68.6 \pm 0.8(984 \pm 44)$ \\
\hline M62 & $39.6 \pm 1.6(389 \pm 15)$ & $63.7 \pm 0.5(793 \pm 13)$ \\
\hline 52100 & $42.6 \pm 0.8(421 \pm 10)$ & $63.0 \pm 1.2(775 \pm 25)$ \\
\hline
\end{tabular}

The hardness of the three PM ingots after heat treatment was 64-69 HRC, consistent with the numbers in the datasheets that Carpenter Technology Corporation provided. The 52100 sample showed comparable hardness to the PM materials.

Microstructures of the materials before and after heat-treatment are shown in Figure 5 and Figure 6, respectively. The $440 \mathrm{C}$ and $440 \mathrm{XH}$ samples showed spherodized carbides, presumably $\mathrm{M}_{23} \mathrm{C}_{6}(\mathrm{M}$ : mainly $\mathrm{Cr}$ ), in the as-received materials which were coarsened after the heat-treatment. The matrix of the $440 \mathrm{C}$ and $440 \mathrm{XH}$ samples after heat-treatment exhibited a bainitic microstructure with a commensurate hardness increase over the as-received material. The M62 sample showed spherodized carbides, presumably $\mathrm{M}_{6} \mathrm{C}$ (M: mainly $\mathrm{Mo}$ and $\mathrm{Fe}$ ), with almost no size and morphology changes before and after the heat-treatment. The matrix of the M62 sample exhibited slight changes in the etched contrast after the heat-treatment, although it was difficult to identify the microstructural feature with the resolution of optical microscope. The microstructure of as-received 52100 material showed a typical air-cooled microstructure consisting of perlite in the matrix together with primary cementite precipitated on the grain boundary. However, after heat-treatment, the microstructure consisted of a martensite matrix with partially transformed perlite (typical oil-quenched microstructure), which showed the formation of spherodized particles in the perlite.

It should be noted that all PM materials show what appears to be some microporosity in the as received HIP'ed condition. This apparent microporosity remains in fully heat treated material but in reality are due to small carbides or small oxides being removed during polishing of the micro samples. The HIP process, with the temperatures and pressure applied to the powder always produces a $100 \%$ dense structure. This condition has no effect on the performance of the alloys.. 


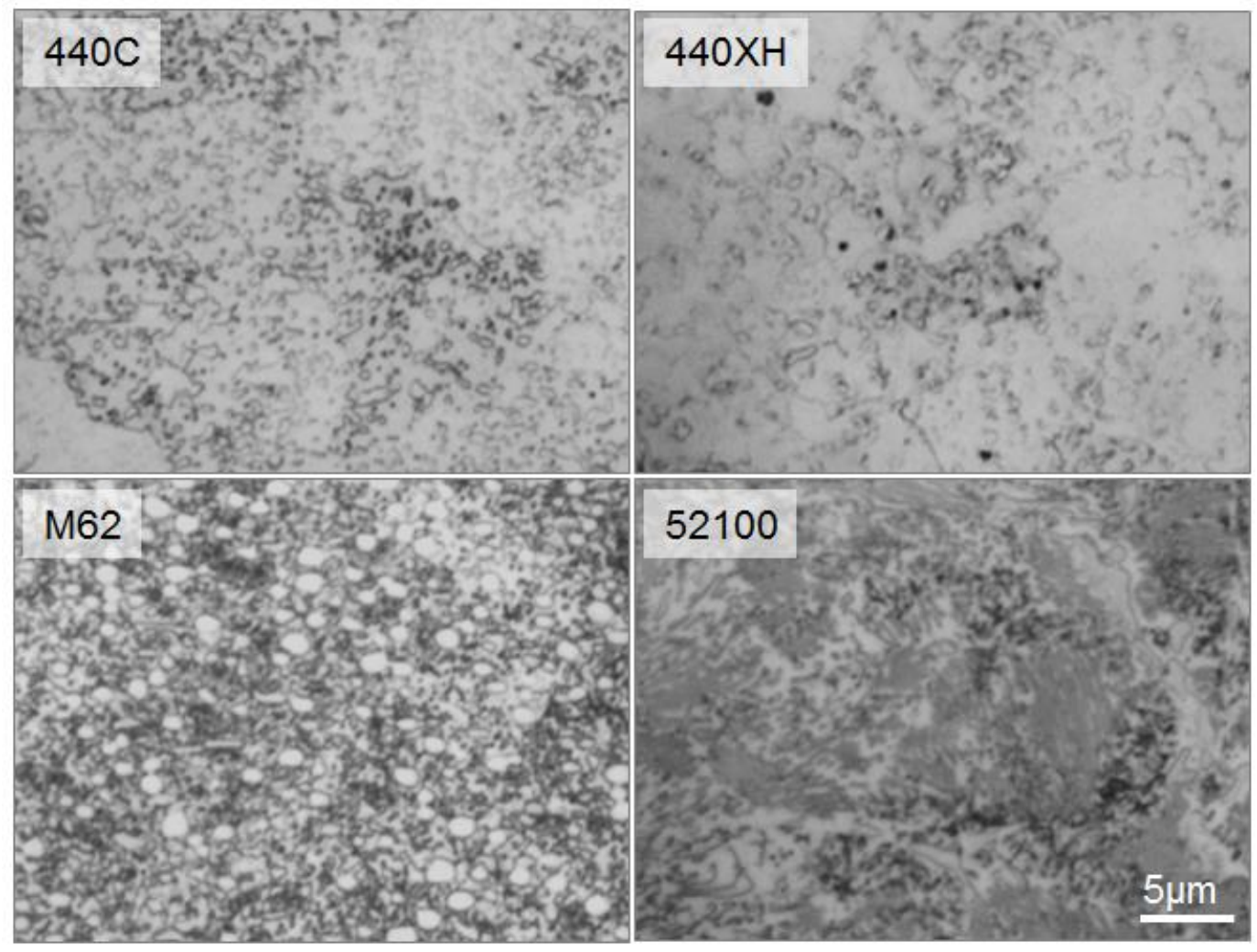

Figure 5. Optical micrographs of the as-received materials.

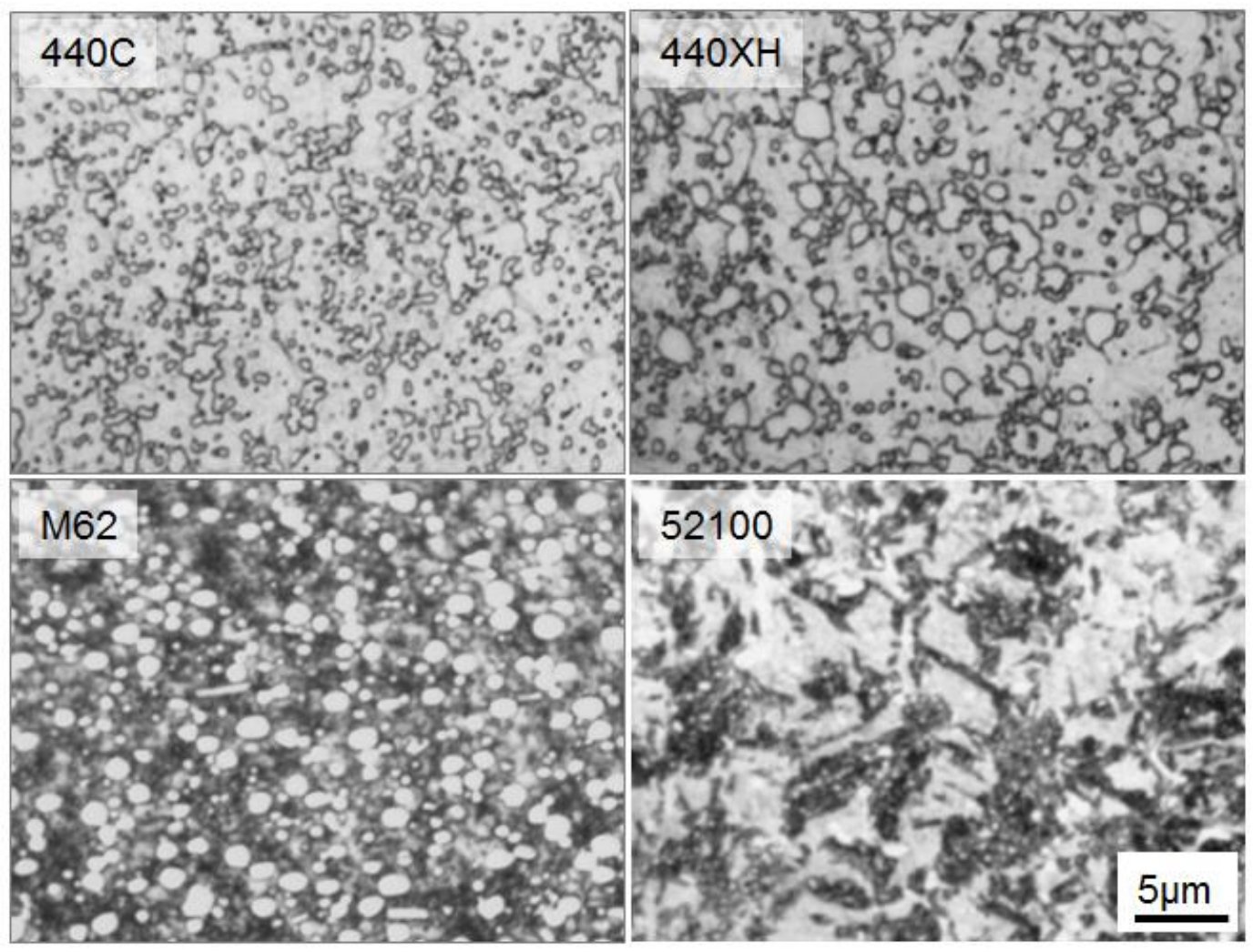

Figure 6. Optical macrographs of the heat-treated materials. 
Further microstructural characterization of the hardened and tempered specimens was conducted by using scanning electron microscopy (SEM) and energy dispersive X-ray spectroscopy (EDS). The $440 \mathrm{C}$ and $440 \mathrm{XH}$ steels showed similar microstructures containing at least two different secondphases as shown in Figure 7a and Figure 7b, respectively. SEM-EDS analysis revealed that the dark contrast phases are Cr-rich carbide, possibly $\mathrm{M}_{7} \mathrm{C}_{3}$ or $\mathrm{M}_{23} \mathrm{C}_{6}$, and the light gray contrast phase are Cr-, $\mathrm{Fe}-$, and Mo-rich, possibly $\mathrm{M}_{23} \mathrm{C}_{6}$ or $\sigma-\mathrm{FeCr}$ phase. Note that the formation of $\sigma-\mathrm{FeCr}$ is not consistent with the literature [1]. A detailed phase analysis such as X-ray diffraction would be required to positively confirm the carbide stoichiometry but is beyond the scope of this work. The M62 in Figure 7c shows three different phases; bright contrast spherical precipitates (Fe-, Mo-, and W-rich, possibly $\mathrm{Fe}_{2}[\mathrm{Mo}, \mathrm{W}]$-Laves phase), bright plate shape precipitates (Mo-rich, possibly $\mathrm{Mo}_{2} \mathrm{C}$ ), and gray contrast precipitates (Fe-, Mo-, V-, and Si-rich, possibly $\mathrm{M}_{6} \mathrm{C}$ ). All three PM samples showed a second phase pull out porosity less than $500 \mathrm{~nm}$ in size and a very small volume percent. The 52100 steel in Figure 7d showed a duplex microstructure with a typical martensitic microstructure (light gray matrix) and fine perlite microstructure (dark gray area) containing many spherodized particles with less than $1 \mu \mathrm{m}$ size, likely $\mathrm{Fe}_{3} \mathrm{C}$ cementite.
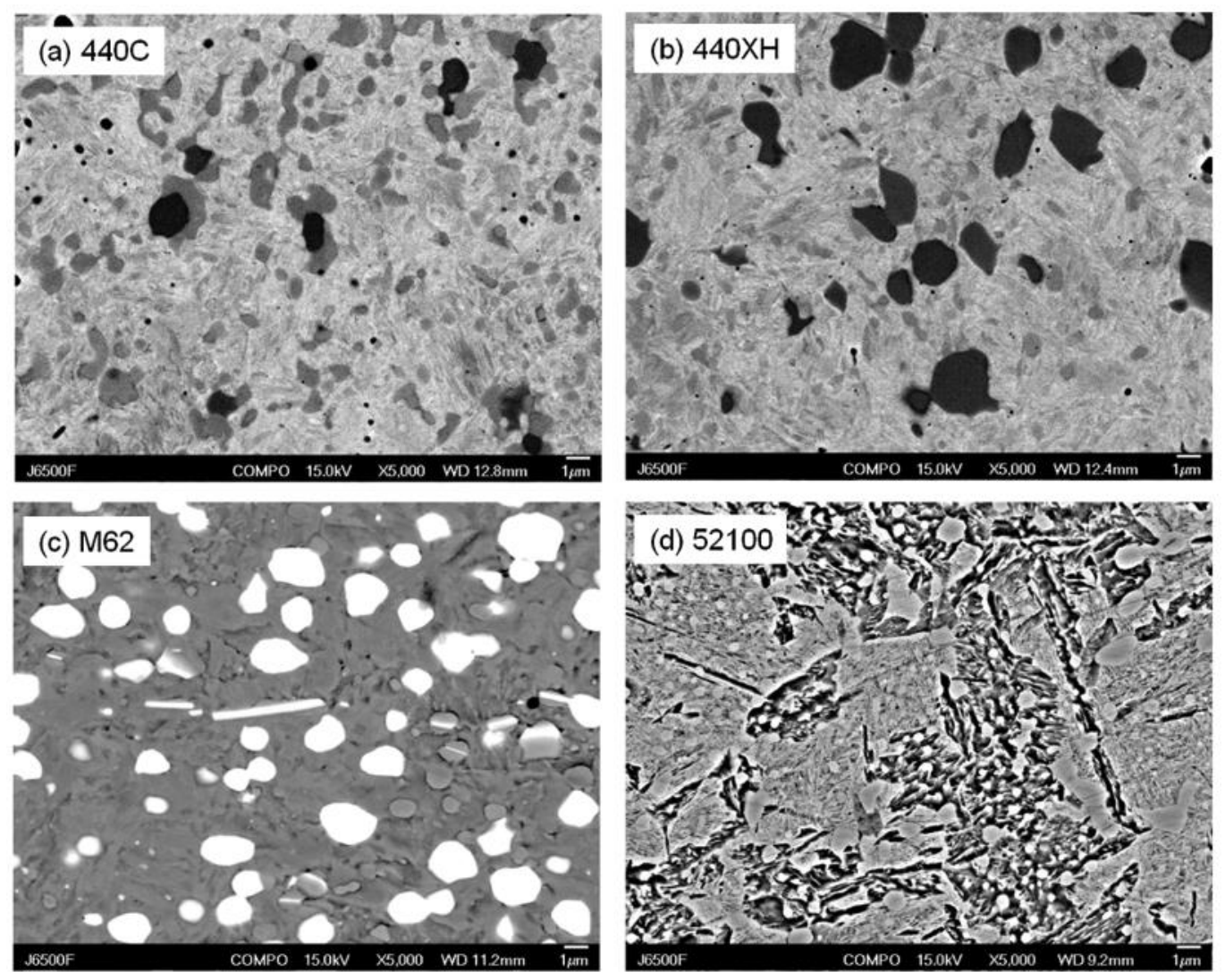

Figure 7. SEM-back scattered electron images of the hardened and tempered specimens. 


\subsection{COMPONENT FABRICATION}

The micro-pitting specimens were machined from five different materials. Figure 8 shows the drawing of the specimen, and a picture of the rough-machined specimens. Since the materials were too hard to be machined after hardening (and tempering) heat-treatments, the rough machining was conducted in the as-received (normalized) condition. The rough machined samples had $0.4 \mathrm{~mm}$ of excess material left to account for final grinding. After the heat-treatment, the samples were ground to final size.
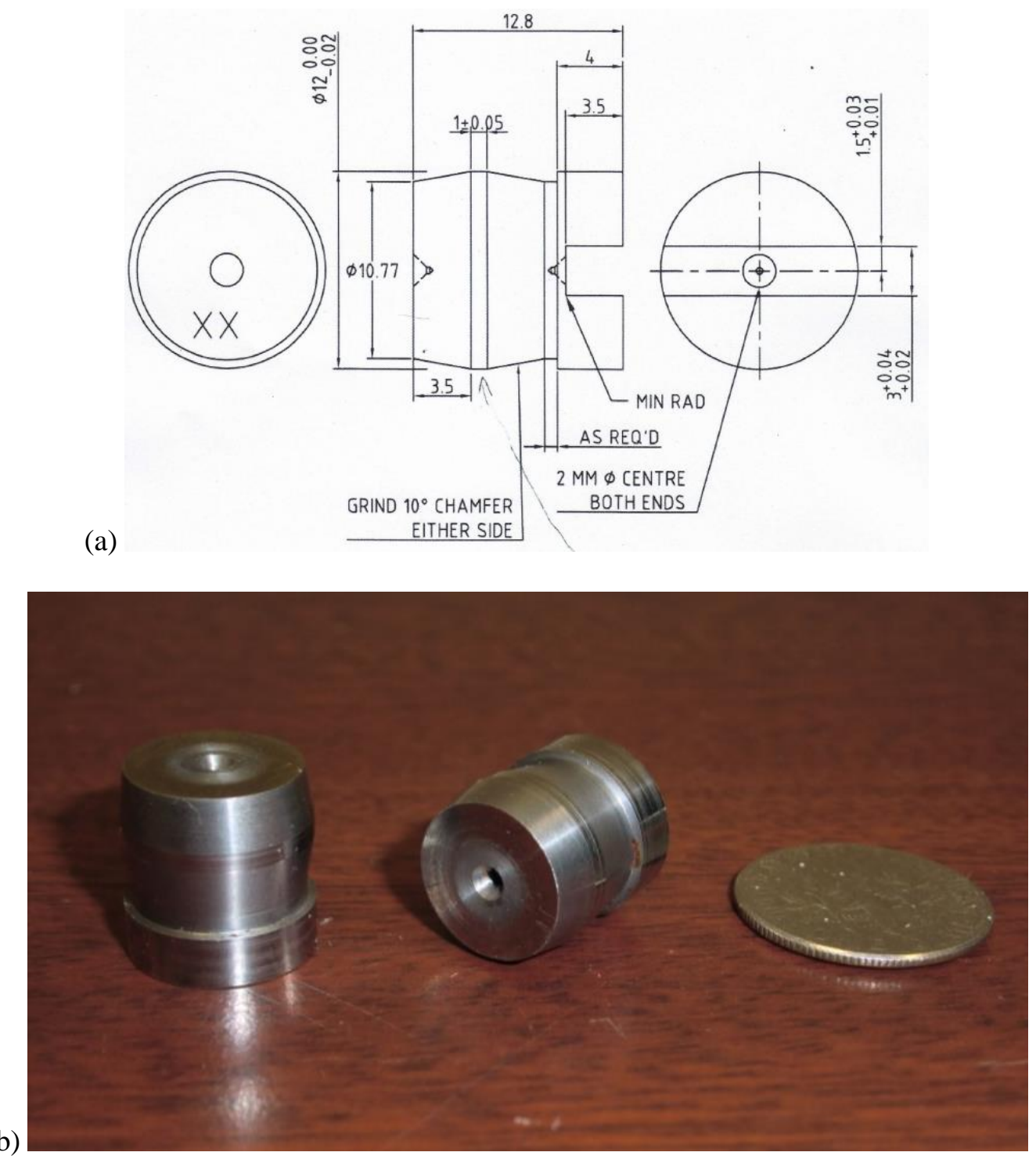

Figure 8. (a) Drawing of the test specimen, and (b) specimens after rough machining.

The hardness of the samples before and after the heat-treatment is summarized in Table 5. There was no significant difference between the hardness of the small sample heat treatments (Table 4) and the specimen heat treatment. The sample after final machining is shown in Figure 9. 
Table 5. Hardness of the samples before and after heat-treatment

\begin{tabular}{ccc}
\hline \multirow{2}{*}{ Alloy } & \multicolumn{2}{c}{ Hardness Rockwell C } \\
\cline { 2 - 3 } & as received & heat-treated \\
\hline $440 \mathrm{C}$ & $40.6 \pm 0.9$ & $64.0 \pm 0.3$ \\
\hline $440 \mathrm{XH}$ & $41.0 \pm 1.0$ & $64.3 \pm 0.4$ \\
\hline M62 & $39.6 \pm 1.6$ & $65.9 \pm 0.4$ \\
\hline 52100 & $42.6 \pm 0.8$ & $60.0 \pm 0.4$ \\
\hline M50 & $40.2 \pm 1.0$ & $66.4 \pm 0.2$ \\
\hline
\end{tabular}

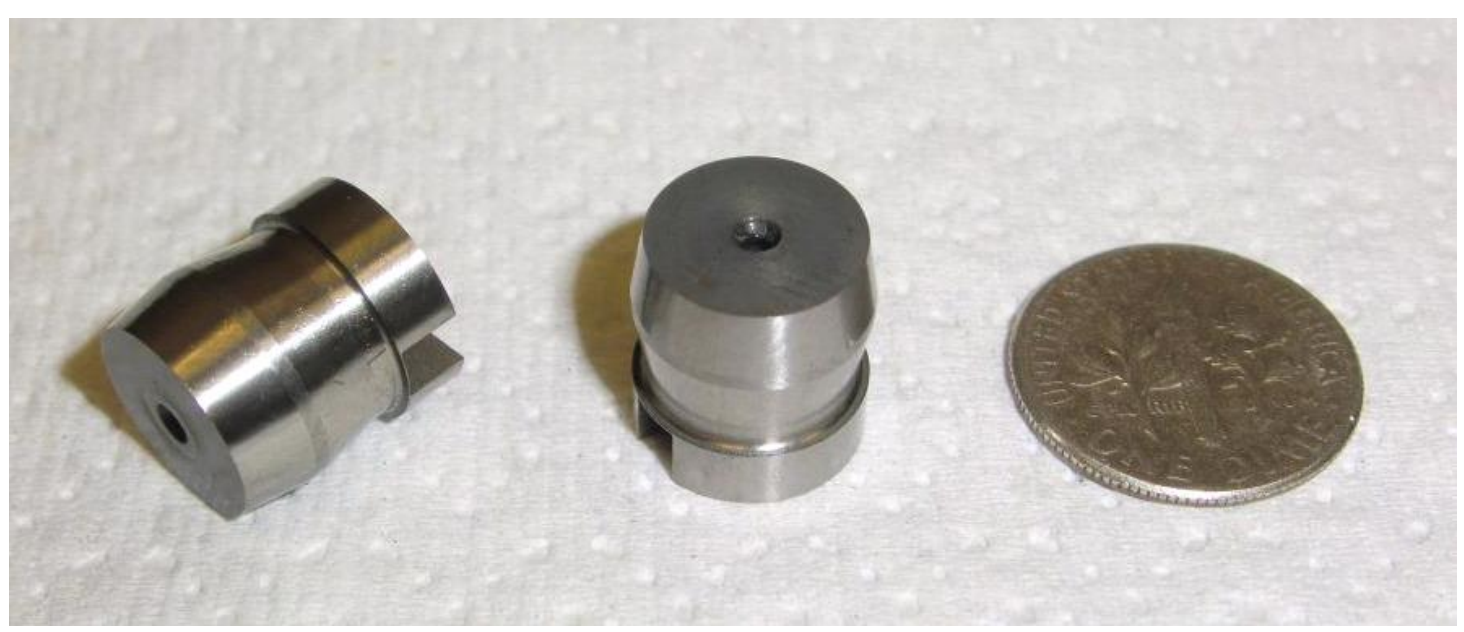

Figure 9. The test specimen (440XH) after final machining.

\subsection{MICRO-PITTING TESTING}

\subsubsection{First Attempt of Micro-pitting Test}

\subsubsection{Test protocol}

Micro-pitting is an acknowledged form of wear damage in wind turbine gear box bearings. A standard definition of the term was recently approved and published in terminology standard ASTM G40-12 [2] as follows:

"micro-pitting, n-in tribology, a form of surface damage in rolling contacts consisting of numerous pits and associated cracks on a scale smaller than that of the Hertz elastic contact semi-width.

DISCUSSION-Micro-pitting features are distinguished from more macroscopic pitting and spalling by the small individual pits (typically a few micrometers to a few tens of micrometers wide). To the naked eye, original metallic bearing surfaces appear duller, giving rise to the term frosting which is commonly used in the bearings literature. Micro-pitting is distinguished from corrosion pitting because fracture is present in each pit."

Micro-pitting may precede more deleterious forms of surface damage, like macro-pitting and spalling, and is therefore of interest to bearing and gear designers. A new micro-pitting test rig from PCS Instruments (UK) was obtained for this project. Experiments using dummy steel specimens were conducted in order to develop a suitable test protocol for the project. In the meantime, a series of twenty test specimens of heat treated steels $(52100, \mathrm{M} 50, \mathrm{M} 62,440 \mathrm{C}$, and 440XH) were precisionmachined to fit the PCS test rig. The specimen and fixture geometry is shown in Figure 3. The 
central roller, approximately $12 \mathrm{~mm}$ in diameter is trapped between three outer disks producing three contact stress cycles for each rotation of the inner roller. The relative speed of the inner roller and disks is chosen to provide a desired amount of slip. Friction, torque, oil temperature and wear are recorded.

A computer program (profile) is generated for each test or portion of each test. This enables tests to consist of a sequence of stages of variable duration. The object is to produce micro-pits on the specimens after a reasonable period of exposure such that the relative resistance to this phenomenon can be determined. Figure 10 shows a micropit developed during procedure development. It was observed after $1.6 \times 10^{6}$ contact stress cycles. Hairline 'tensile' cracks appear perpendicular to the horizontal slip direction that is indicated by the scuffing marks. Coalescing cracks eventually produce loss of material and pits a few micrometers or tens of micrometers across.

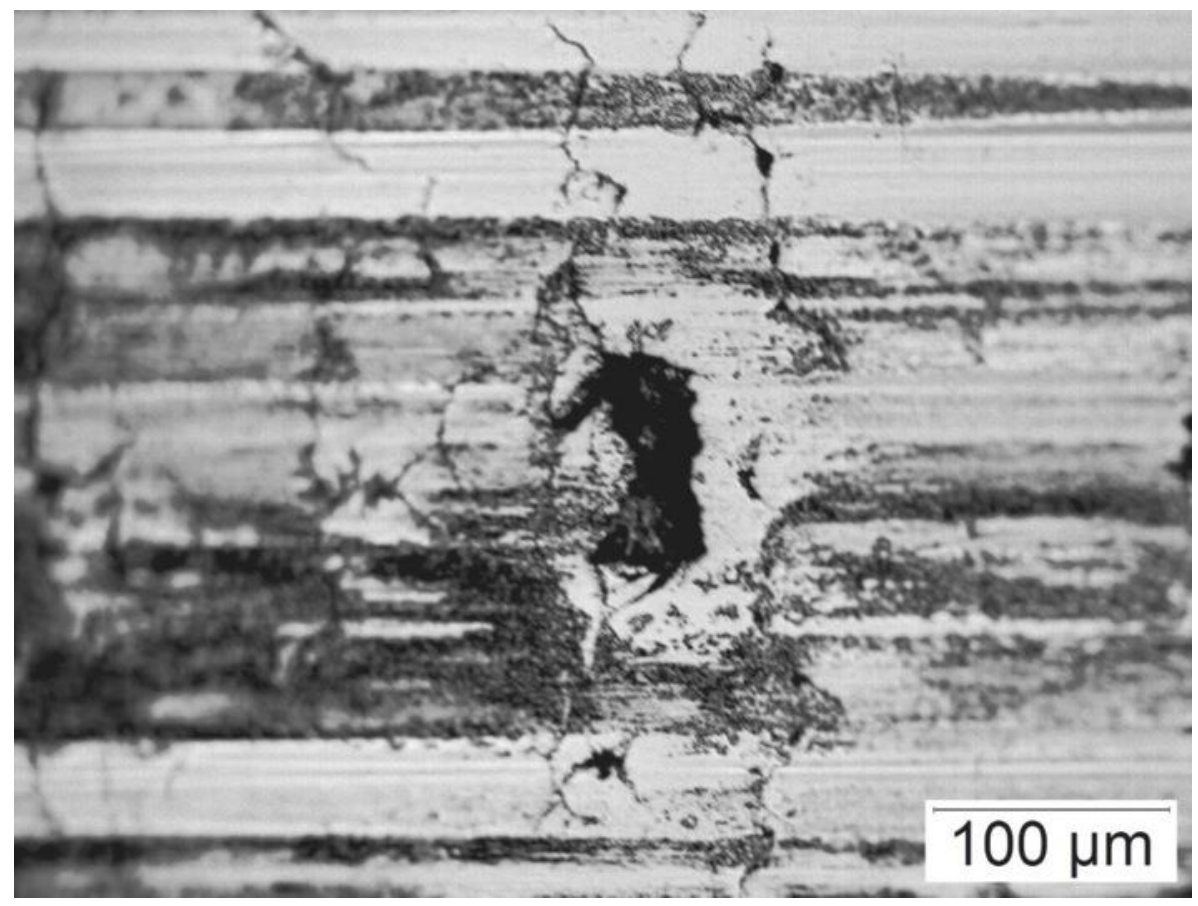

Figure 10. Micropit produced of a steel surface during a preliminary experiment.

All five alloys were micro-pitting tested with the protocol shown in Table 6. There were three different stages to apply the different loads $(230,305$, and $390 \mathrm{~N})$ with a constant rate $(3.0 \mathrm{~m} / \mathrm{s})$ and slip $(20 \%)$. Figure 11 shows the loading steps (loads in kilo-newtons), the corresponding friction coefficient data (Figure 11a), and wear results (Figure 11b) for the first test run on M50 tool steel. Friction coefficient decreases as the surface runs in. The friction coefficient data was averaged in each step, and used for the discussion later in Figure 12. Most wear typically occurs during the first stage of the test because the initial surface runs in and conforms to the counterface. High-spots on the machined roller and disk profiles are quickly worn away. Near the end of the first stage, the wear rate tends to slow down. 
Table 6. Micro-pitting Test Protocol (first trial)

\begin{tabular}{|r|l|c|}
\hline Stage & \multicolumn{1}{|c|}{ Conditions } & Duration \\
\hline 0 & $\begin{array}{l}\text { Run-in: Speed at 3.0 m/s, increase from 0 to 230 N, maintain 0\% slip, room } \\
\text { temperature }\end{array}$ & $5 \mathrm{~min}$ \\
\hline 1 & First load stage: Speed constant at 3.0 m/s, load at 230 N, 20\% slip, room temperature & $60 \mathrm{~min}$ \\
\hline 2 & $\begin{array}{l}\text { Second load stage: Speed constant at 3.0 m/s, load at 305 N, 20\% slip, room } \\
\text { temperature }\end{array}$ & $60 \mathrm{~min}$ \\
\hline- & Remove and examine the test roller for signs of surface damage, then replace & -- \\
\hline 3 & Third load stage: Speed constant at 3.0 m/s, load at 390 N, 20\% slip, room temperature & $60 \mathrm{~min}$ \\
\hline- & Remove and examine the test roller for signs of surface damage & -- \\
\hline 4 & $\begin{array}{l}\text { Fourth stage: same load, speed, and slip conditions as for third stage, but adding 2.5 } \\
\text { hours of additional running time to generate more wear (run on selected specimens) }\end{array}$ & $150 \mathrm{~min}$ \\
\hline
\end{tabular}

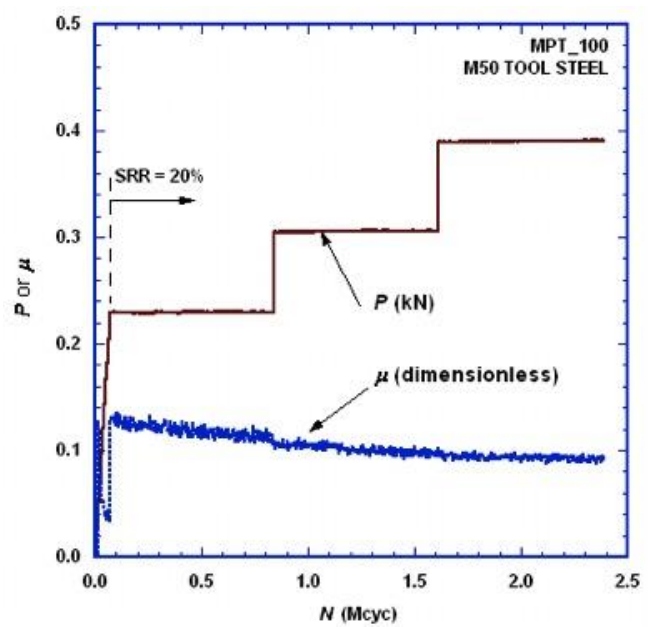

(a)

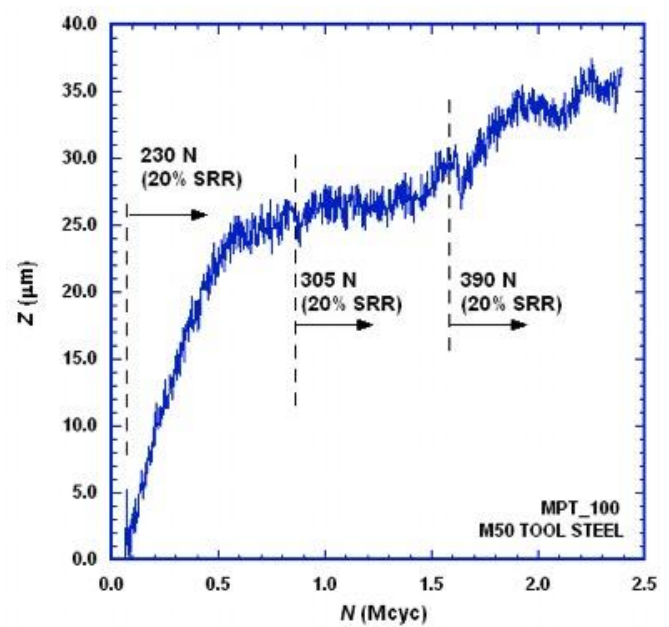

(b)

Figure 11. (a) Load history, corresponding friction coefficient, and (b) wear results for M50.

\subsubsection{Test results (first attempt)}

Figure 12 summarizes the friction data for all ten micro-pitting tests (repeats for each alloy). All alloys survived the full protocol, and the automated shut-off controls, based on excessive vibration or temperatures exceeding $135^{\circ} \mathrm{C}$ did not activate. Therefore, none of the alloys exhibited the kind of catastrophic micro-pitting, which would trigger a premature shut-down. The friction coefficients are reported as mean values for each load stage. With a few exceptions, the friction coefficient tends to decrease for each load stage for the five alloys. The relative influence of material properties for the rolling/sliding couple on the friction coefficient is dependent on a combination of factors such as the lubricant properties, the regime of lubrication, and the state of surface roughness. The latter effects are summarized by a parameter called the film thickness to roughness ratio $(\Lambda)$. This is defined as the ratio of the minimum lubricant film thickness divided by the root-mean-square of the arithmetic average roughness $(\mathrm{Ra})$ of opposing surfaces. As temperature increases, the viscosity of the lubricant decreases, leading to a thinner film and the likelihood of more incidental surface contact, $(\Lambda$ decreases). From this point of view, the higher the friction coefficient, the more likely that the materials were playing a role in its magnitude. Friction coefficients in the range of 0.08 to 0.2 (typ.) are in the boundary lubrication regime, suggesting that both the lubricant and materials contributed to the friction coefficient. Also note that as wear leads to an increase in surface roughness, the $\Lambda$ ratio decreases, promoting solid-solid contact and accelerating the degradation. Within the scatter of $\mu$ data, no alloy appeared significantly better than the others. 


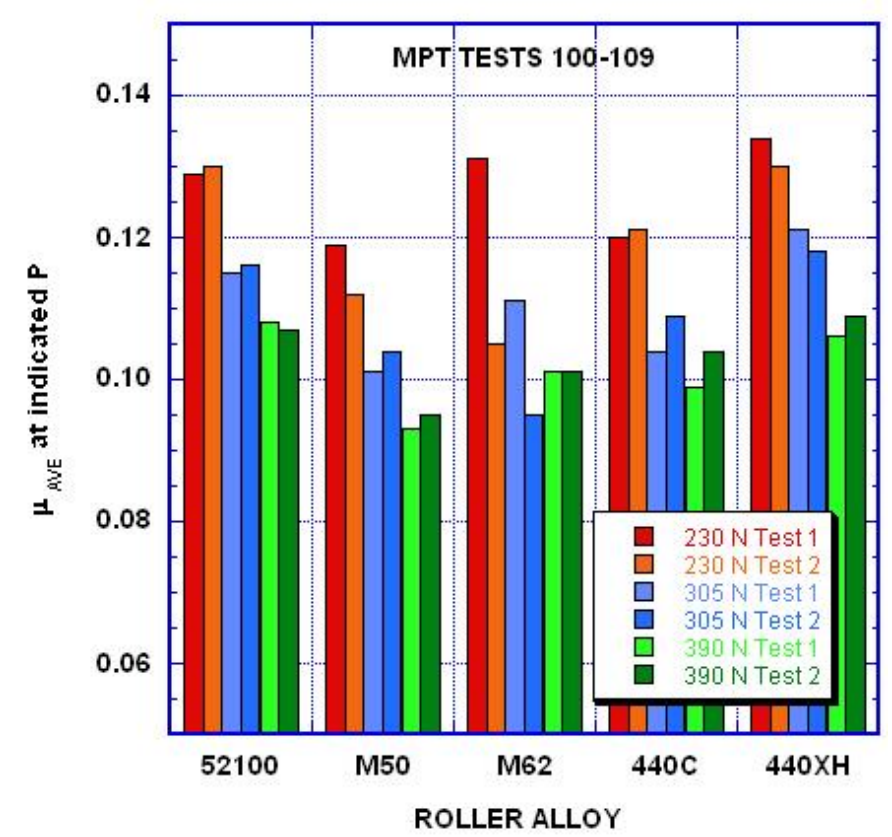

Figure 12. Average friction for each loading step for two tests of each alloy.

Figure 13 summarizes the wear for stages 1 and 2, for stage 3, and for the entire test, given in terms of specimen displacement, $\mathrm{z}(\mu \mathrm{m})$. Most displacement of the roller/disk couple, associated with wear and conformity of the interface, occurred during the earlier stages of the test despite the lower applied loads. This indicates that running-in of the initial surface finish dominated the wear during the first part of the tests. The 440XH had least initial wear, suggesting that its higher hardness tended to retard the effects of initial wear-in. Note also that there was a greater degree of consistency in $\mathrm{z}$ for duplicate tests in Stages 1 and 2, and a much larger variability in repeated tests for Stage 3.

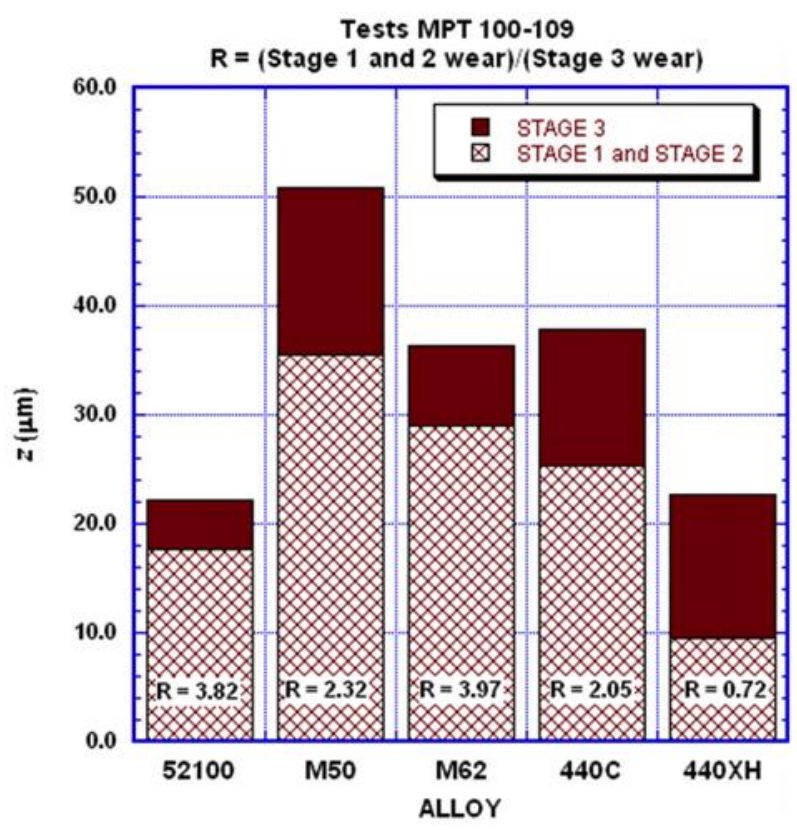

Figure 13. Wear summary for two tests of each alloy (average wear from 2 runs). For all but the 440XH with its higher hardness, the running-in wear dominated Stages 1 and 2 and wear in Stage 3 was lower. 
In micro-pitting tests, wear occurs by the development and loss of small spalls of material that may not significantly result in surface recession. As debris is lost, the load is transferred to the remaining material and most of the bearing surface remains. From displacement data alone, one would conclude that the 52100 and $440 \mathrm{XH}$ had the best performance; however, quantifying wear only by the displacement of the surfaces may be problematical for the reasons described, and such results should be combined with observations of the damage as well. Furthermore, considering the need to clean the specimens of residual lubricant and the potential for debris embedment, it is impractical to measure wear of micro-pitting specimens by weight loss.

Table 7 summarizes the wear data of the alloys in terms of displacement. Two tests were conducted in each material, and one of them was used for microstructure characterization by interrupting the test after stage 3. Figure 14 shows the wear data comparison of (a) the test-interrupted samples for microstructure characterization, and (b) the samples continued to stage 4. During stages 1 and 2 (which correspond to the lower loading test steps with 230 and $305 \mathrm{~N}$, respectively), the displacement seems consistent in each material. The $440 \mathrm{XH}$ showed minimum displacement among the samples, the 52100 followed, and the M50 showed maximum displacement, in both Figure 14a and Figure 14b. However, after stage 3 with $390 \mathrm{~N}$ loading, the displacement significantly varied in the same materials. The smaller displacements tend to show the material dependence similar to the displacement in stages 1 and 2 (Figure 14a). These samples were used for microstructure characterization. On the other hand, the larger displacements after stage 3 do not show any reasonable relationship with the displacements in stages 1 and 2 (Figure 14b). Further continuous testing in stage 4 with the same loading resulted in a similar tendency to that in stage 3 . It is still unclear whether this reflects the material nature or experimental errors, and it needs to be addressed by conducting additional testing with a different condition.

Table 7. Wear data of the alloys (in terms of displacement, $\mu \mathrm{m}$ ) for micro-pitting testing.

\begin{tabular}{|c|c|c|c|c|c|c|c|}
\hline Alloy & ID & $\begin{array}{c}\text { Stages } 1+2 \\
(60+60 \text { min })\end{array}$ & $\begin{array}{c}\text { Stage } 3 \\
\text { (60 min) }\end{array}$ & $\begin{array}{c}\text { Total wear } \\
\text { (1 through } \\
3)\end{array}$ & $\begin{array}{c}\text { Stage } 4 \\
(+150 \mathrm{~min})\end{array}$ & $\begin{array}{c}\text { Total wear } \\
\text { (1 through 4) }\end{array}$ & Remarks \\
\hline \multirow{2}{*}{52100} & 102 & 20.3 & 8.4 & 28.7 & 25 & 53.7 & \\
\hline & 103 & 15 & 0.7 & 15.7 & - & - & Test interrupted \\
\hline \multirow{2}{*}{ M50 } & 101 & 40 & 23.1 & 63.1 & 36 & 99.1 & \\
\hline & 100 & 31 & 7.4 & 38.4 & - & - & Test interrupted \\
\hline \multirow{2}{*}{ M62 } & 107 & 31 & 7.7 & 38.7 & 10 & 48.7 & \\
\hline & 106 & 27 & 6.9 & 33.9 & - & - & Test interrupted \\
\hline \multirow{2}{*}{$440 \mathrm{C}$} & 104 & 25.4 & 20.5 & 45.9 & 35 & 80.9 & \\
\hline & 105 & 25.5 & 4.3 & 29.8 & - & - & Test interrupted \\
\hline \multirow{2}{*}{$440 \mathrm{XH}$} & 108 & 9 & 24.8 & 33.8 & 40 & 73.8 & \\
\hline & 109 & 9.9 & 1.6 & 11.5 & - & - & Test interrupted \\
\hline
\end{tabular}


(a) Test interrupted after stage 3 (for characterization)

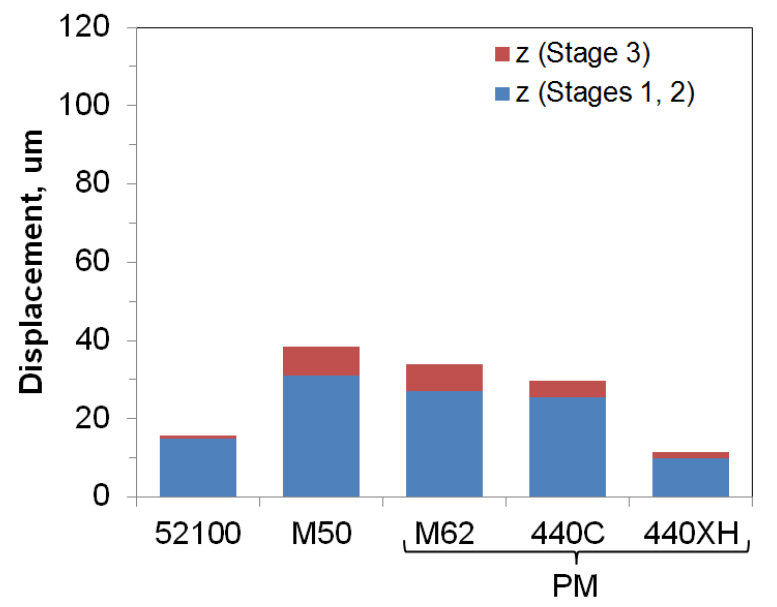

(b) Test continued to stage 4

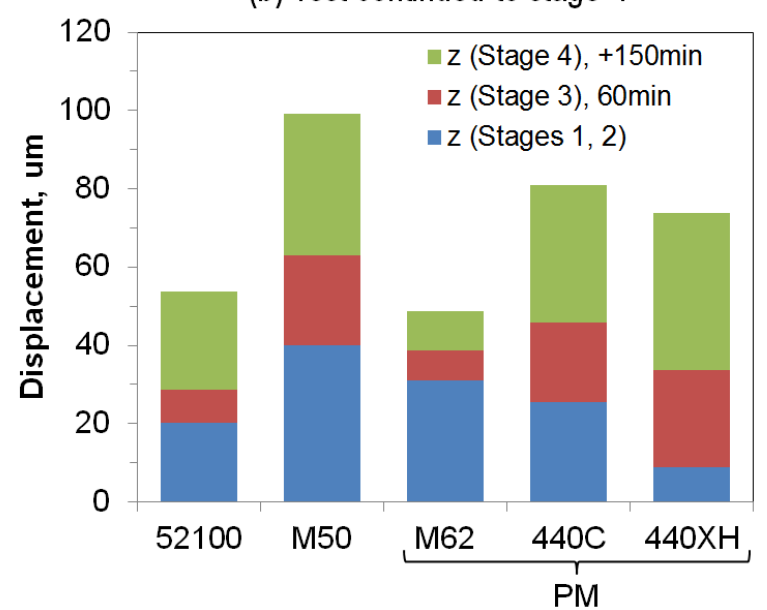

Figure 14. Wear data comparison of (a) the test-interrupted samples for microstructure characterization and (b) the samples continued to stage 4.

\subsubsection{Microstructure characterization}

Microstructure characterization of the tested samples after stage 3 was completed. The specimen surface and cross-sectional view were observed by SEM (scanning electron microscopes). Figure 15 shows a picture of the sample together with the indications of the observed portions. A narrow band at the sample ridge corresponds to the tested surface contacted to the alloy steel disks, and the slopes in both sides of the band correspond to the untested surface. The cross-sectional samples were prepared after completing the surface observation. Figure 16 shows the SEM-SE (secondary electron) images of the sample surfaces, with and without micro-pitting testing. The vertical axis corresponds to the rolling/slipping (tested surface) and machining (untested surface) directions. Figure 17 shows the SEM-BSE (back-scattered electron) images of the cross-sectional views of the samples, in the same orders of those in Figure 16.

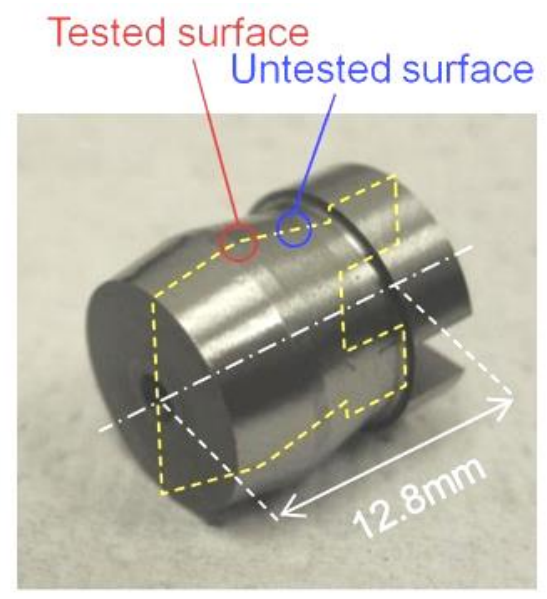

Figure 15. A picture of the sample $(440 \mathrm{XH})$ before testing.

The roughness of as-machined (untested) surfaces of the 52100 and $440 \mathrm{XH}$ seems very high, the 440C follows, and the M62 and M50 show less roughness. This indicates that the roughness would 
depend on the hardness of the materials. However, from the cross-sectional views, the gap of the surface relief between top and bottom is less than $3 \mu \mathrm{m}$, so that it is almost negligible compared to the displacement after stage 3 (11.5 to $38.4 \mu \mathrm{m})$. All tested surfaces became relatively flat compared to the untested surfaces. The M62 and 440XH showed smeared features on the tested surfaces, indicating that they were plastically deformed. However, there was no significant change in the crosssectional microstructure near the surface. All PM materials showed cracks inside the matrix after testing, which were initiated from the second-phases or the interphase boundaries. Most of the cracks propagated parallel to the surface, indicating that there may be a work-hardened layer on the tested surface which would fail to cause micro-pitting. Previous macroscopic observation found the micropitting feature only on the 52100 and M62. However, no apparent relationship between the wear resistance and microstructure was observed.

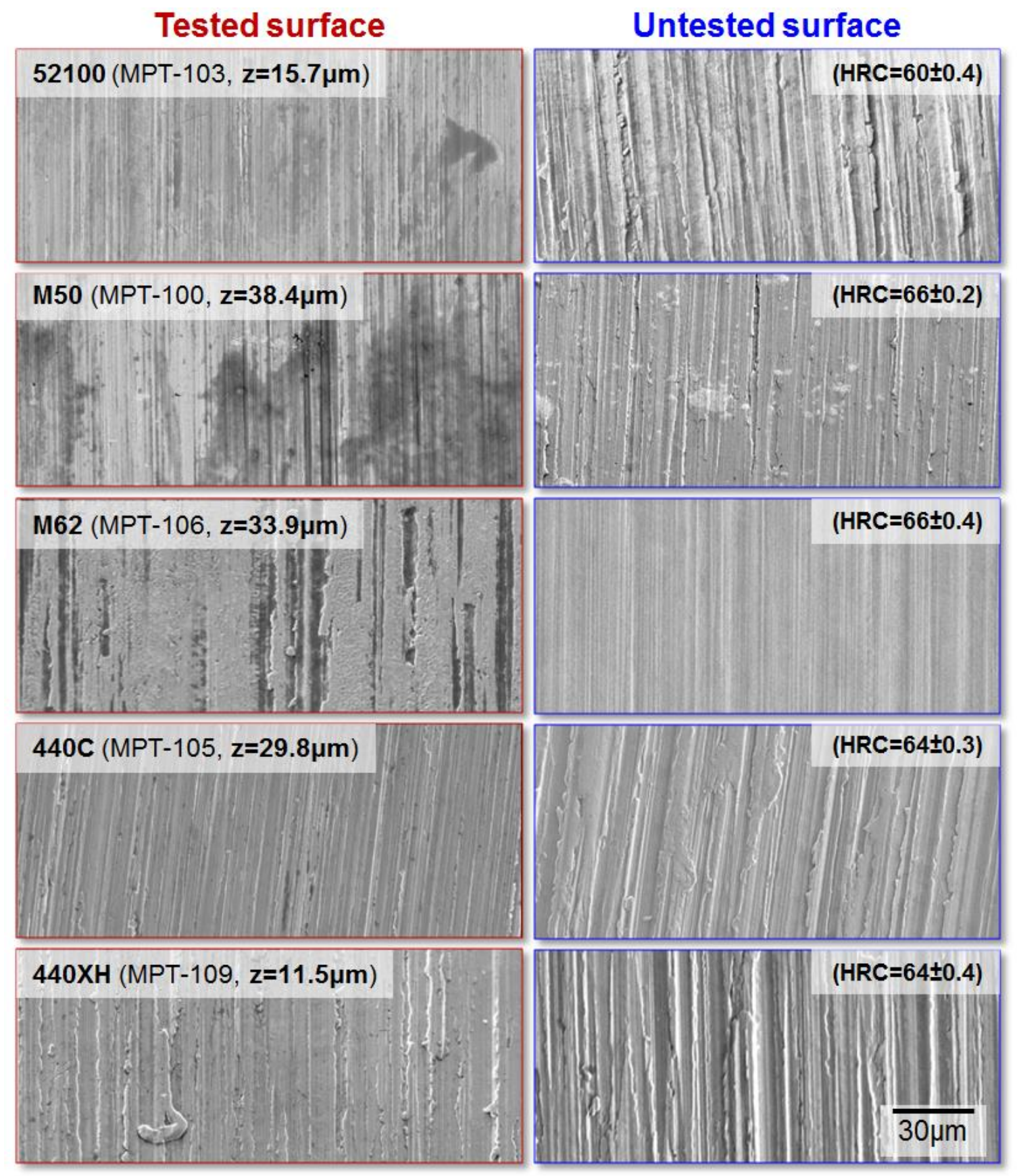

Figure 16. SEM secondary electron images of the sample surface. 
Most of the tested surface observation suggested that the major wear mode of the present samples was plastic deformation only near the tested surface, which could be due to high slip ratio (20\%). In order to observe the micro-pitting-type failure, the test condition needs to be less slip ratio (2-5\%) with higher loading, which would give a fatigue on the contact surface to promote micro-pitting.

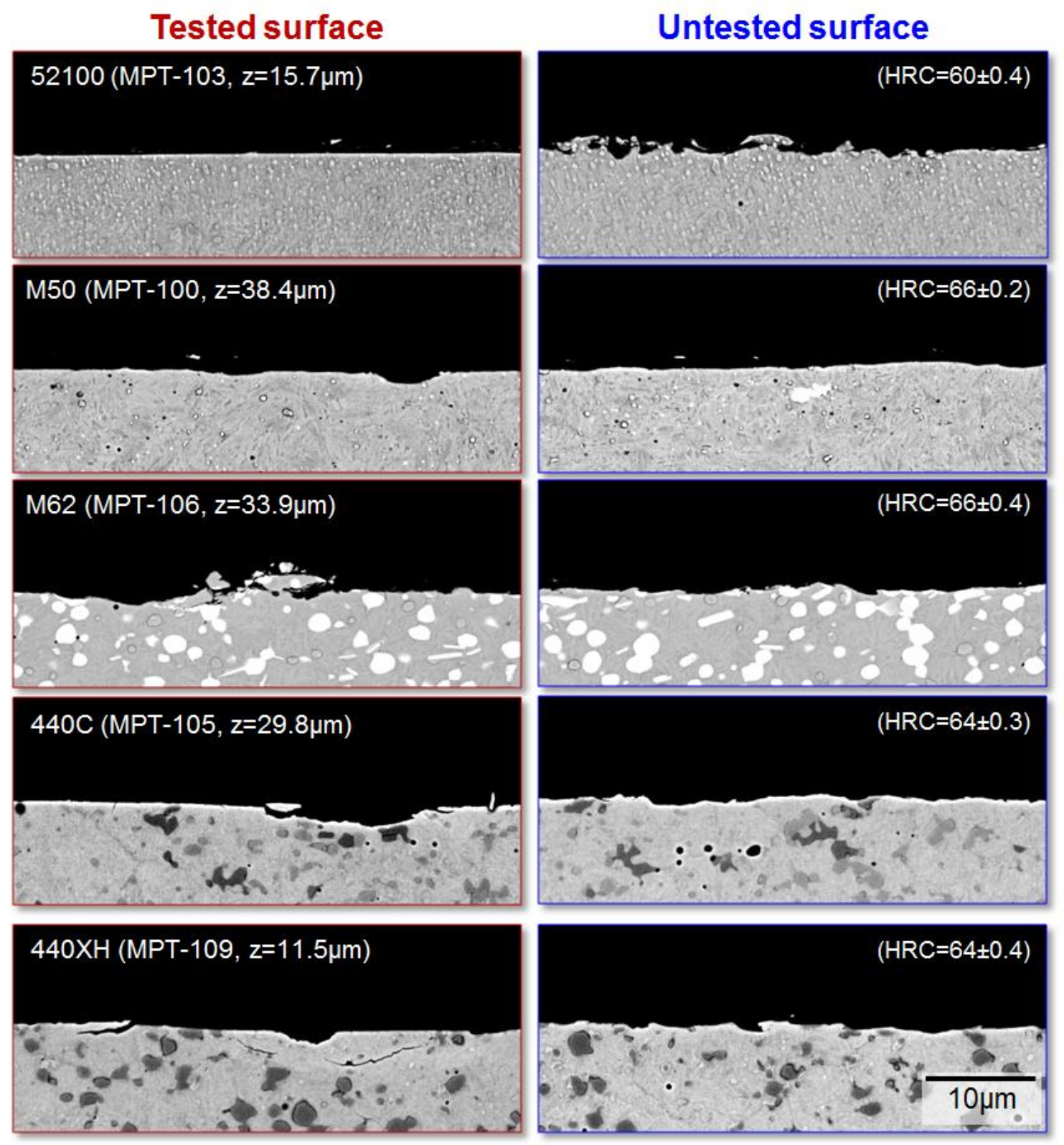

Figure 17. SEM back-scattered electron images of the cross-sectional view.

\subsubsection{Optimized Test Conditions Dedicated Micro-pitting Formation}

In order to observe micro-pitting features on the samples, optimized test conditions with higher load and less slip were applied. Table 8 lists the programmed loading sequence that was used in this work. Requiring 10 minutes, stages A1-3 were designed to provide an initial run-in up to full load. After 
examination to ensure stable operation, the test was restarted and run up to full load (Stages B1-2). Each roller received a total of 6.7 million contact cycles during the test.

Table 8. Micro-Pitting Test Protocol (second trial)

\begin{tabular}{|l|c|c|c|c|c|}
\hline \multicolumn{1}{|c|}{ Parameter } & Stage A1 & Stage A2 & Stage A3 & Stage B1 & Stage B2 \\
\hline \hline Initial speed (m/s) & 3.5 & 3.5 & 3.5 & 3.5 & 3.5 \\
\hline Final speed (m/s) & 3.5 & 3.5 & 3.5 & 3.5 & 3.5 \\
\hline Initial load (N) & 0 & 230 & 650 & 0 & 650 \\
\hline Final load (N) & 230 & 650 & 650 & 650 & 650 \\
\hline Initial contact stress (GPa) & 0 & 1.289 & 2.133 & 2.133 & 2.133 \\
\hline Final contact stress (GPa) & 1289 & 2133 & 2133 & 2133 & 2133 \\
\hline Initial SRR (\%) & 0 & 5 & 5 & 5 & 5 \\
\hline Final SRR (\%) & 0 & 5 & 5 & 5 & 5 \\
\hline Duration (min:ss) & $1: 00$ & $2: 00$ & $7: 00$ & $1: 00$ & $390: 00$ \\
\hline Cumulative cycles (roller) & $1.671 \times 10^{4}$ & $5.013 \times 10^{4}$ & $1.671 \times 10^{5}$ & $1.838 \times 10^{5}$ & $6.701 \times 10^{6}$ \\
\hline
\end{tabular}

The minute weight loss of material produced in micro-pitting (MP) makes measurement of MP more subjective than quantitative, and experimenters often relying on either detecting an increased level of vibrations or visual examinations of surfaces rather than mass loss or wear depth, as are common in quantifying other forms of wear and surface damage. Subjective assessments like YES/NO micropitting, or the number of cycles until the vibration sensors automatically shut down the testing machine have been used. Attempts were also made to qualitatively assess the degree of micro-pitting using terms like 'not observed', 'minor micro-pitting', 'moderate micro-pitting', and 'severe micropitting' or 'spalling'. While not precise, these provide more information than a simple 'yes or no.' In the present work, a hierarchy of damage features was defined, and the specimens were photographed to compare the relative amounts of micro-pitting and other wear features between the tested steels.

In this work, micro-pitting was accompanied by a variety of other damage features, some of which directly concern micro-pit formation and growth, and others that suggest abrasive wear and adhesive wear. This combination of surface damage processes, and their relative influence depends upon such things as the slide/roll ratio, the lubricant type, the micro-and macro-geometry of contact, and of course the characteristics of the materials. Cumulative time or numbers of contact cycles witness changes in the competition between surface damage processes. For example, running-in and wear-out represent a transition from one set of interfacial processes to another [3]. Therefore a system of categorizing various features on test rollers was used.

\subsubsection{Classification of damage}

Figure 18 depicts a proposed hierarchy of damage modes arranged by relative severity, several of which were observed in this study. Those designated by $\mathrm{S}$ involve sliding contact and those designated by $\mathrm{P}$ involve pitting. Micro-pitting and non-micro-pitting phenomena can occur together and on the same surface. Each is associated with four increasing levels of severity. Level S1 involves isolated asperity micro-scale deformation and truncation and is a precursor to $\mathrm{S}$ that involves gross polishing or light scuffing, causing the surface roughness to decrease for a period of time. This is actually a positive aspect in reducing micro-pitting because the number of sharp, localized stress concentrators decreases. Higher slide/roll ratios may therefore wear surfaces smoother and thus retard micro-pitting. Abrasive wear (S3) can be either two-body, if the counterface has hard protrusions or three-body if loose material (wear debris) is produced from micro-pits. An example of abrasive wear grooves on the M-50 tool steel surface is shown in Figure 19. There were few identifiable micro-pits, but a preponderance of abrasion marks. At level S4, one observes deep gouging and scoring of surfaces, possibly from larger spalled particles. Galling or seizing level is unlikely except for very high sliding components and high loads where the lubricant film is wholly 
inadequate. Levels P1-P2-P3 reflect features observed in this work. Level P4 is associated with more gross failures, such as those described elsewhere for spherical roller-type gearbox bearings [4].

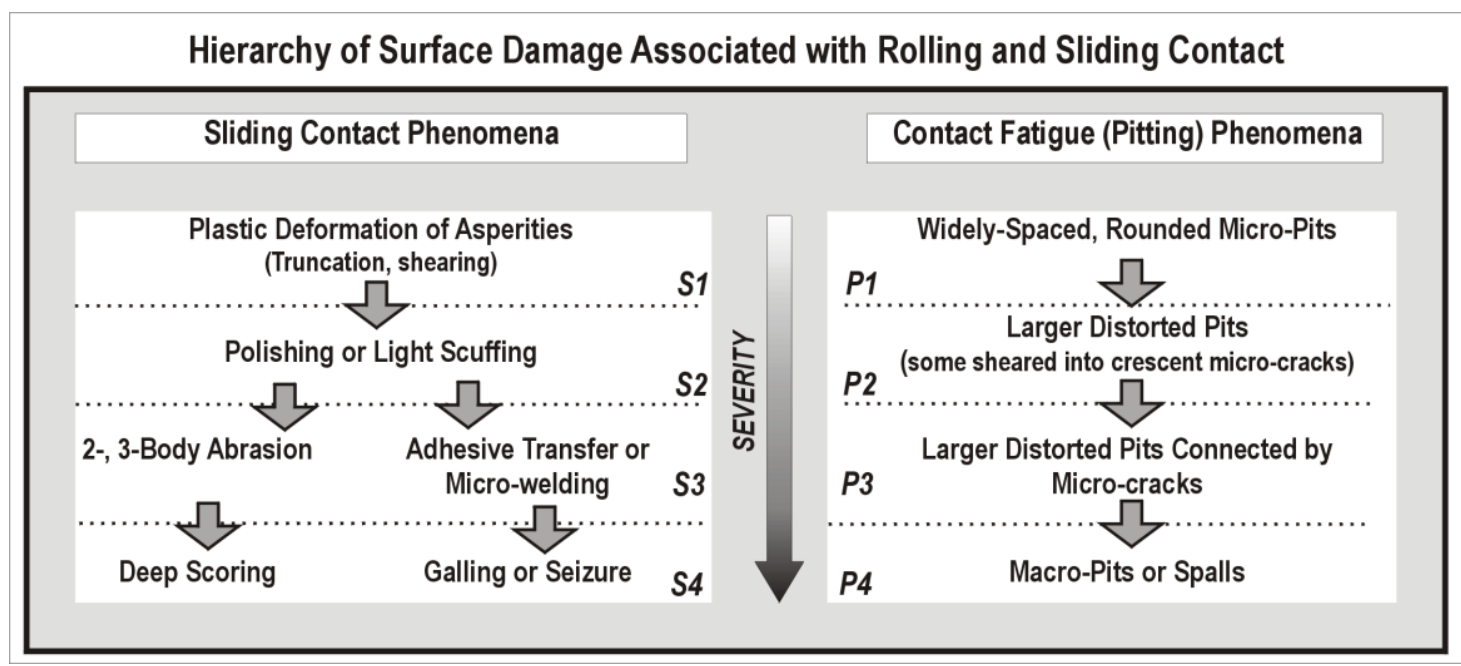

Figure 18. Hierarchy of surface features observed on metallic surfaces subjected to a combination of rolling and sliding.

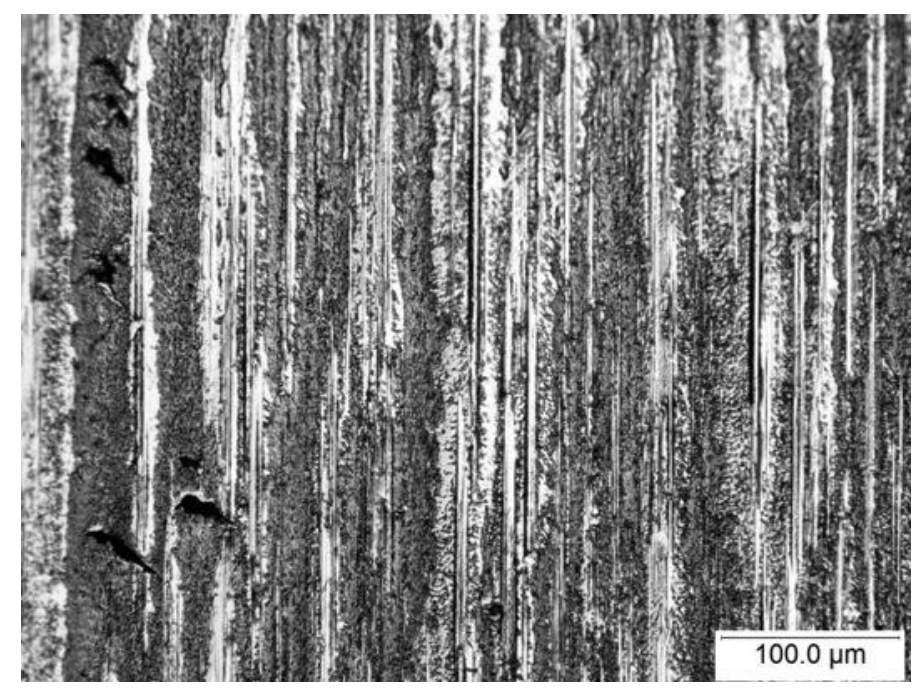

Figure 19. Abrasive wear grooves and a few micro-pits on the surface of an M-50 steel roller.

\subsubsection{Microstructural observations}

An example of a relatively new pit that has not yet been distorted by plastic shear, is shown in Figure 20a for a 440C steel roller. The rim of the pit seems smooth and polished, perhaps because it stood higher than the surrounding surface after the material inside the pit was pulled out. In comparison, most of the micro-pits observed in this work were distorted or sheared closed in a crescent shape, such as those shown in Figure 20b for M50 tool steel. Note the prominent abrasive groove entering from the center right in Figure 20b that seems to produce displacement of the right side rim of the pit almost to touch the opposite side. Also in Figure 20b some of the displaced material from the pit can be seen overhanging the horizontal groove that lies below the pits, indicating that this material was displaced after the groove was formed. The micro-fracture coalescence mechanism of pit formation is indicated by the brick-wall like network of defects at the bottom of a pit in 52100 steel made visible after the overlying material was lost (Figure 21a). The manner in which cracks can extend from the maximum depth of a micro-pit to the free surface at each edge can be seen in the oblique view of a 
micro-pit from the same roller in Figure 21b. Corresponding cross-sectional views of these defects in 52100 are shown in Figure 22.

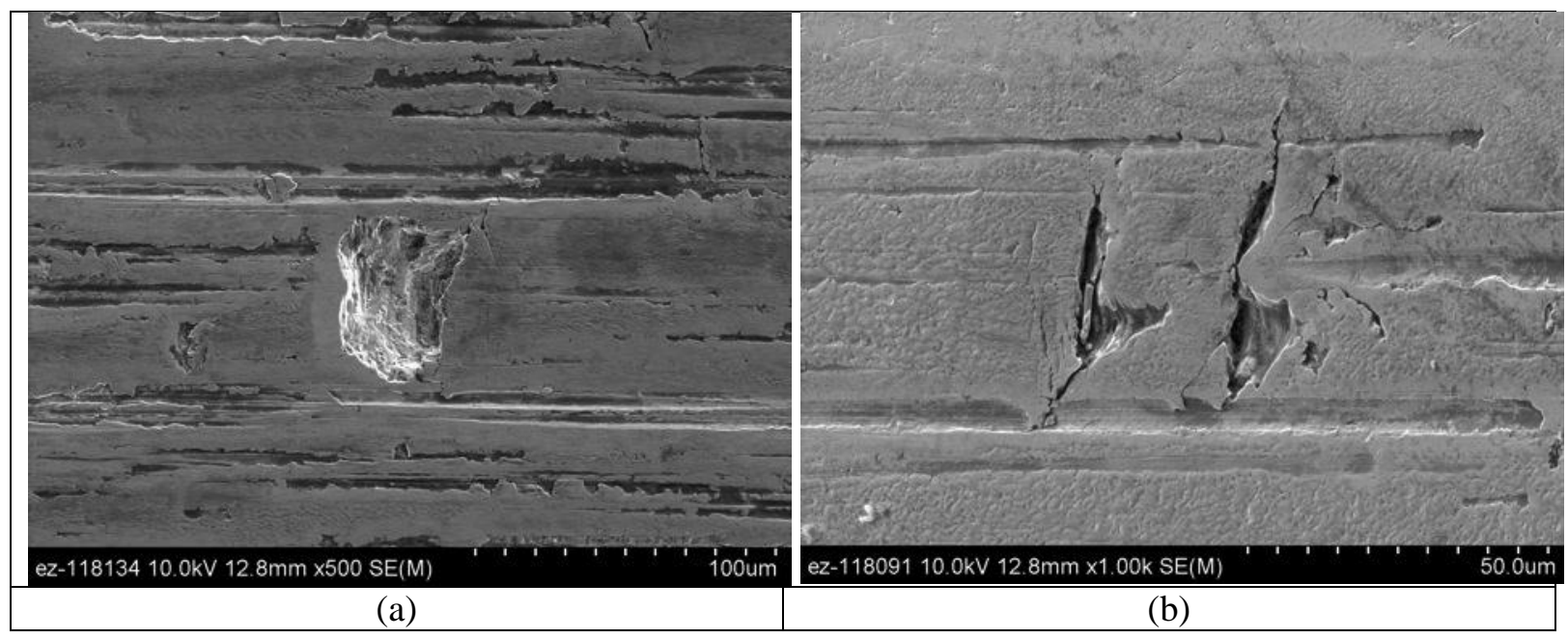

Figure 20. (a) A rounded micro-pit in 440C steel, and (b) distorted micro-pits in M50 tool steel where shear has partly or fully closed them, leaving fine cracks at both ends.
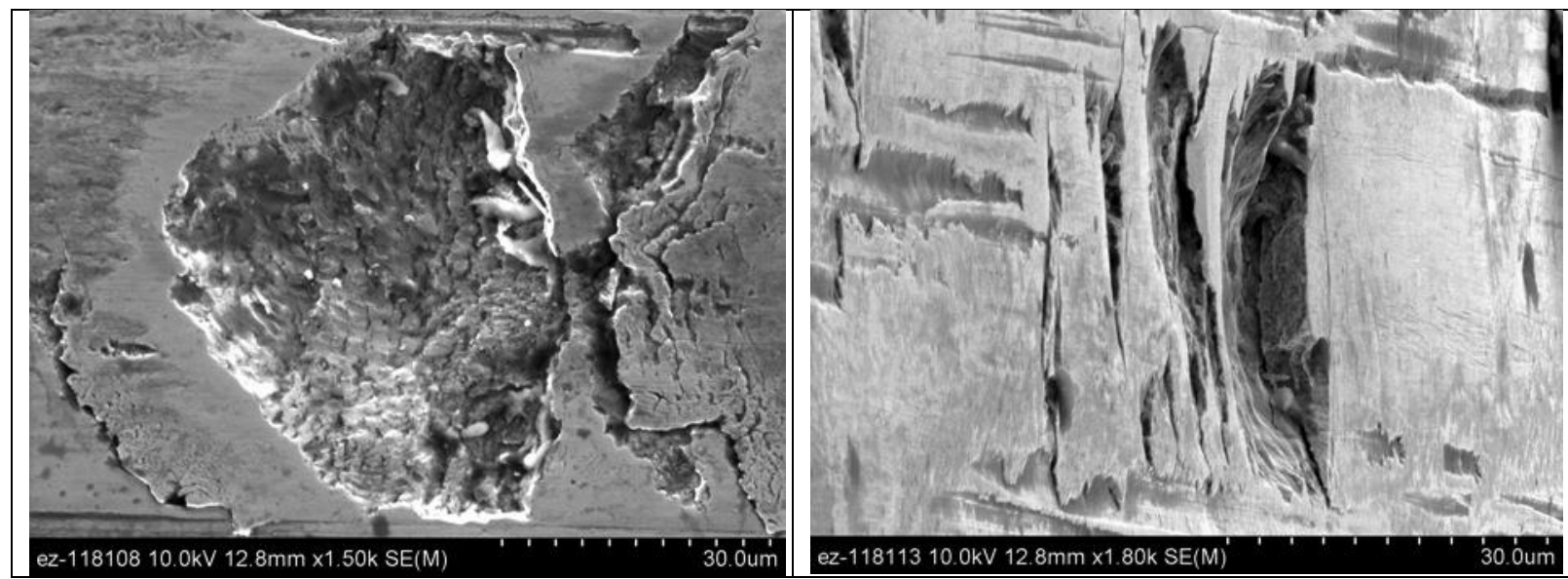

Figure 21. Micro-pit in 52100 showing (a) a blocky network of micro-cracks, and (b), an oblique view of micro-pits in the same steel specimen showing the arc-like shape of a micro-crack that underlies a shelf of material, as seen at the right in the cross-section in Figure 7(a).

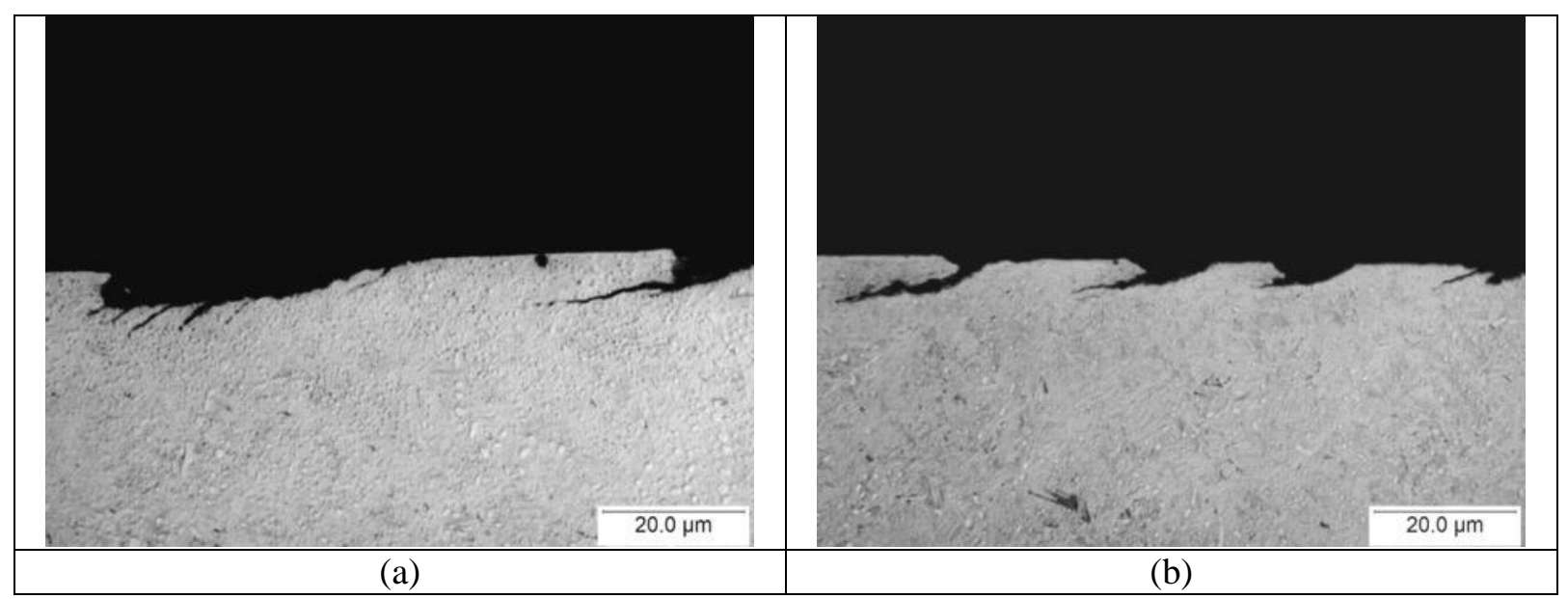


Figure 22. Polished and etched (2\% nital) cross-section of the 52100 steel roller showing (a) a series of micro-fractures extending from the bottom of a micro-pit like that shown in Figure 21(a), and (b) a row of micro-cracks underlying the surface. The open end of one such crack can be seen in Figure 21(b). The slip direction was right to left, since the pits open in the tensile field following passage of the contact patch.

In examining the steels, of which 52100 showed the most extensive MP-related damage, the features revealed a direct interaction between sliding (abrasion)-related phenomena and rolling-related mechanisms. For example, a flap of material partially covering a micro-pit could be loosened by fatigue to the point where a passing asperity from the counterface or a loose particle of debris could remove it by abrasion.

Like any metal fatigue process, micro-pitting is thought to consist of a nucleation period during which subsurface damage accumulates, then crack initiation, crack propagation, and eventual material loss when cracks break the surface and linkup (see Figure 23). The interior walls of micro-pits in several of the alloys contained evidence for fatigue and for the presence of branched networks of microcracks that consolidate below the surface prior to the loss of a debris particle that eventually forms a pit. A piece of material about to be lost to form a new pit is shown in Figure 24.

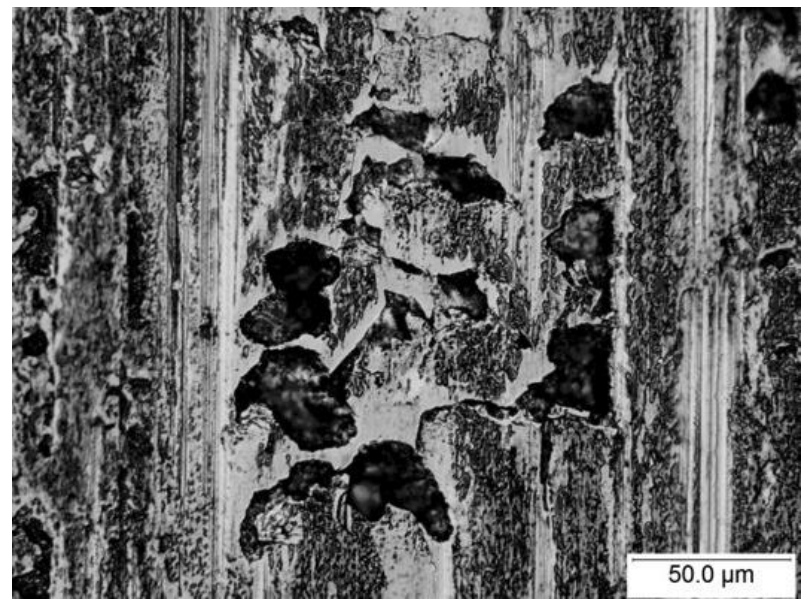

Figure 23. Series of micro-pits on $\mathbf{5 2 1 0 0}$ steel that are connected by micro-cracks. The widths and interior scratches within the abrasive grooves are similar in size to the breath of the micro-pits suggesting that wear debris from the pits can serve as third-body abradants.

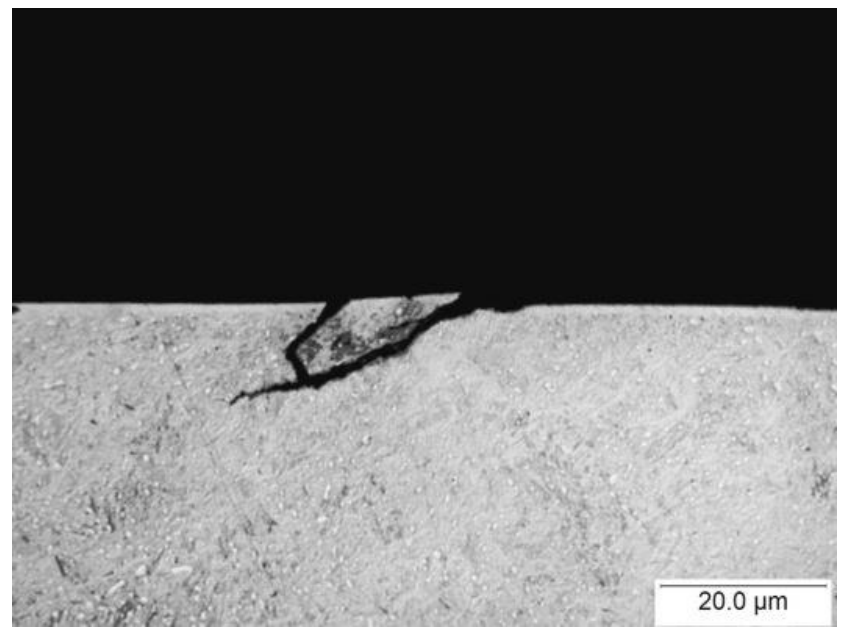

Figure 24. Particle of $\mathbf{5 2 1 0 0}$ steel about to leave the surface to leave a micro-pit behind. (2\% nital etch) 
The types of damage on the surfaces of roller specimens, observed using optical microscopy at magnifications of 210 to $420 \mathrm{X}$, were characterized using the hierarchical structure displayed in Figure 18. Results are shown in Table 9. All specimens contained varying degrees of both slidingrelated and rolling (fatigue) related damage, but non showed evidence for P4. Therefore, a column has been added to the right of Table 9 to provide a qualitative assessment of the pitting severity.

Table 9. Categorization of Features Observed on Surfaces of Steel Test Specimens

\begin{tabular}{|l|c|c|c|c|c|c|c|l|}
\hline \multicolumn{1}{|c|}{ Alloy } & S1 & S2 & S3 & S4 & P1 & P2 & P3 & Observations \\
\hline \hline 52100 & $\mathrm{X}$ & $\mathrm{X}$ & $\mathrm{X}$ & & & $\mathrm{X}$ & $\mathrm{X}$ & $\begin{array}{l}\text { severe connected } \\
\text { MP, local scuffing, } \\
\text { abrasion }\end{array}$ \\
\hline M50 & $\mathrm{X}$ & & $\mathrm{X}$ & & $\mathrm{X}$ & & & $\begin{array}{l}\text { limited MP, mainly } \\
\text { significant abrasion }\end{array}$ \\
\hline M62 & & $\mathrm{X}$ & $\mathrm{X}$ & & & & & $\begin{array}{l}\text { limited MP, mainly } \\
\text { significant abrasion }\end{array}$ \\
\hline 440C & & & $\mathrm{X}$ & & $\mathrm{X}$ & $\mathrm{X}$ & & $\begin{array}{l}\text { few areas of MP } \\
\text { near edges }\end{array}$ \\
\hline 440XH & & & $\mathrm{X}$ & & $\mathrm{X}$ & & & least MP \\
\hline
\end{tabular}

The 52100 steel showed the most severe indications of MP, and the pits were beginning to link up, possibly to produce larger spalls (e.g. see Figure 23). In contrast, the $440 \mathrm{XH}$ alloy, showed less evident micro-pitting (e.g. see Figure 25). Alloy 440C was also less MP prone, but there were some areas near the edges of the rollers with minor MP. Interestingly there seemed to be no direct relationship between Rockwell $\mathrm{C}$ hardness and MP tendency. Both the softest (52100, HRC 60) and the hardest (M50, HRC 66) alloys displayed more incidence of MP than the stainless steels which had intermediate hardness ( HRC 63-64). Carbon content did not seem to correlate with MP either, but the most obvious compositional difference in the steels is that the two 400-type martensitic stainless steels, with their high $\mathrm{Cr}$ contents, performed best overall. The $440 \mathrm{XH}$ grade contains a finer and more uniform microstructure and that may be one reason why MP nucleation was less prevalent.

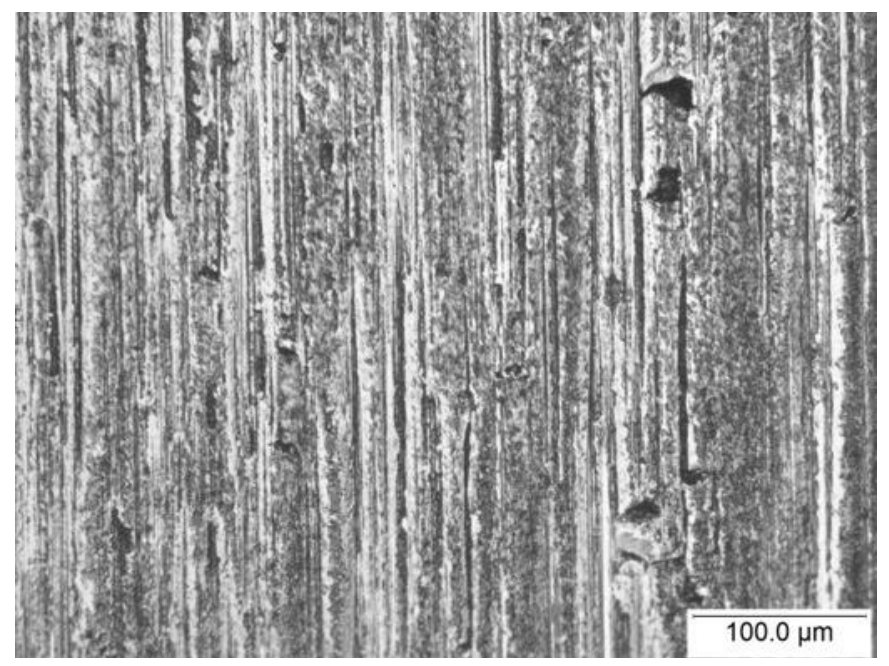

Figure 25. Reduced frequency and size of micro-pits in alloy 440XH.

A tribosystem of the type studied here involves two counter-surfaces of dissimilar composition: the roller and the rings; therefore it is worth noting some observations concerning the ring specimens as well as the rollers. The ring specimens were made intentionally smoother than the rollers to focus micro-pitting on the latter, but there was also evidence of surface breaking cracks on the ring 
specimens. For example, the ring used for the 52100 steel which micro-pitted significantly tended to have S3 level abrasive scratches and grooves, but with no clear indications of micro-pitting. In contrast, the test rings used against M-50 steel retained their polished appearance, but did show surface-breaking micro-cracks (Figure 26).

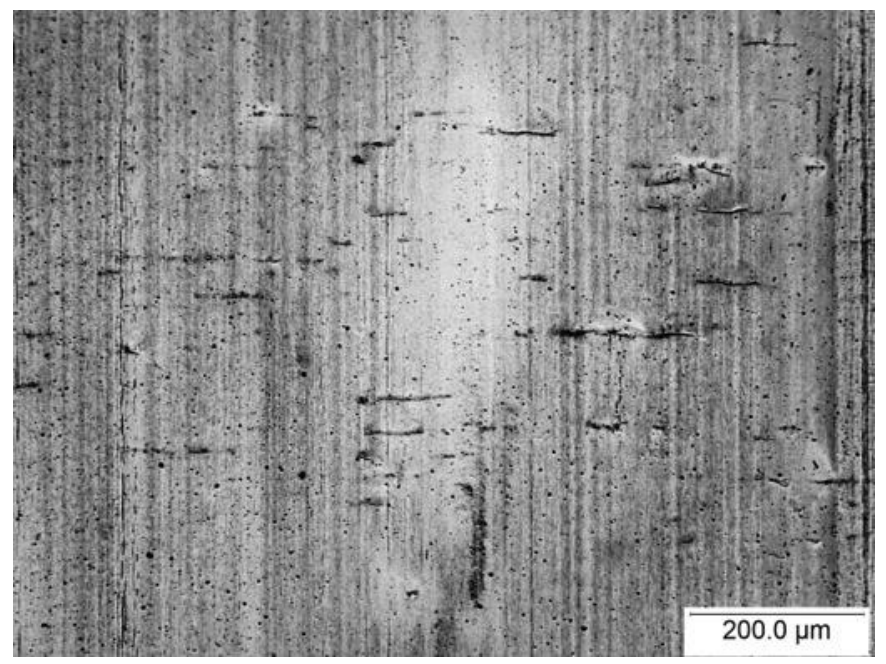

Figure 26. Hairline, transverse cracks suggestive of micro-pitting initiation in the ring specimen used with M-50 rollers.

Clearly, opposing rolling element surfaces such as those used in the current work experience comparable loading histories, and in the current work, the authors acknowledge that contact damage was not exclusive to the roller specimen. However, the emphasis here was on characterizing the five steels used for rollers, and a detailed characterization of the disks was not undertaken.

\subsubsection{A note on white layers and micro-pitting}

In recent years there has been increased interest in the formation of what have been called 'white layers' in the zone below contact surfaces (e.g., $[5,6,7,8,9,10])$. Features that appear white or nearly featureless in polished and etched cross-sections have been observed in conjunction with wear for many years, especially on ferrous materials and in heavily-loaded applications like railroad rails, gear teeth, and highly-stressed rolling elements. Their appearance can vary depending on the materials involved, the severity and geometry of surface contact, and even the type of etchants used to reveal them. In prior work on worn wind turbine bearings [4], relatively thick white layers were easily visible, and many contained networks of fine cracks associated with spalls and other surface damage features (e.g., see Figure 27). In the present work, however, etched cross-sections of the test specimens revealed no clear evidence of such layers although there are localized areas in which the near-surface microstructures of the rollers were distorted and sheared (e.g, see Figure 22). High magnification optical examination of the most micro-pitting resistant steels (440C and 440XH) revealed micro-cracks emanating below micro-pits that avoided the blocky carbide particles in the microstructure as they propagated through the matrix material (see Figure 28). Note that the microcrack extends below the surface several times the depth of the surface pit, but there is no apparent white layer. Based on the present work, white layers seem less associated with MP, which occurs on the localized asperity level, than with macro-pitting which seems to be associated with more extensive, heavily work-hardened subsurface regions, extending many times the length and breadth of individual features. 


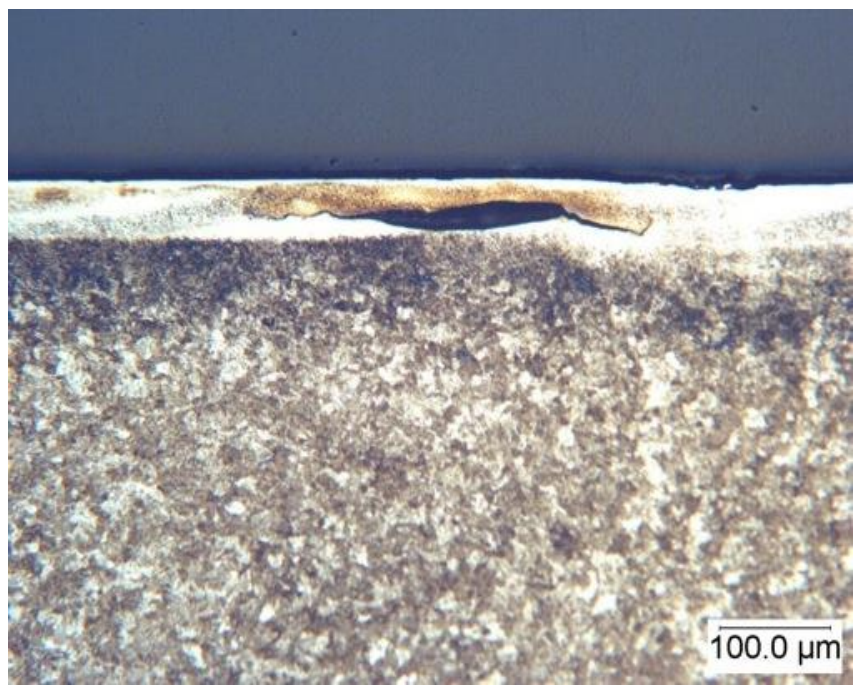

Figure 27. White layer on a bearing steel used in a wind turbine gearbox bearing showing a subsurface void and extending micro-cracks associated with the nucleation of spalls.

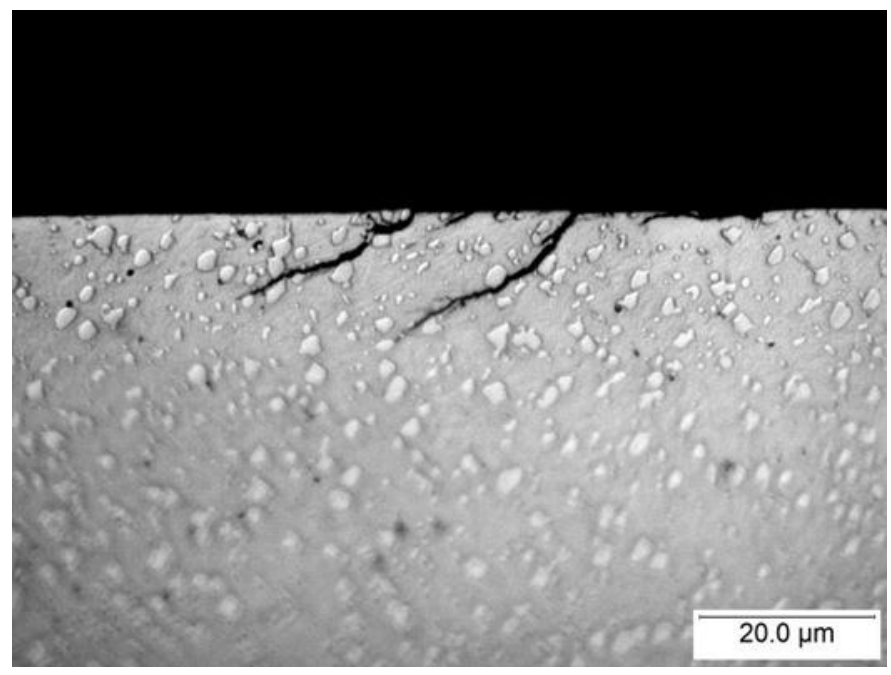

Figure 28. Micro-cracks avoiding carbide particles as they extend from the bottom of a micro-pit. (glyceregia etch) 


\section{BENEFIT ASSESSMENTS}

The benefits in prolonging the life of steel components for energy applications are discussed from two perspectives: 1) loss of steel in wear, and 2) downtime of a renewable energy system (particularly in the area of renewable energy such as wind turbine bearings). The first area of interest for energy assessments is universal and relates to the shortcomings in using steel in wear prone applications without devising mitigation strategies. Often, purchases of materials/components are based on initial costs, and may not capture maintenance, repair, and downtime to develop cost models for full life of system. Steel components, especially steel bearings, are used in many renewable energy systems. Of particular interests are wind turbines where bearings are used throughout the system and their failure can lead to sizeable downtime, component replacement and service costs, and loses in recoverable energy.

Steels are used through a broad range of engineering applications including bearings due to their mechanical properties, availability, and relatively low cost. However, most steels are not optimally designed for wear resistance. Direct and indirect costs and energy losses associated with the wear of steel components are not easily aggregated. One study performed in 1978 attempted to quantify the annual cost to the U.S. economy. Estimated at $\$ 20$ billion annually at the time of the report, this would have the same buying power as $\sim \$ 65$ billion dollars annually today [11xi]. Estimates provided in an ASM handbook from 2001 listed annual energy loses in lost weight of steel due to wear of components in industrial operations, Table 10 [8]. Included in their estimate is 505 TBtus of energy in steel loss due to wear in utilities, 52 TBtus in mining, 19 TBtus in agriculture, and 14 TBtus in primary metal fabrication [8]. Another statistic [8] stated that "Highway vehicles alone use annually $14,600 \mathrm{TBtu} /$ ton of energy represented in lost weight of steel and $18.6 \%$ of this energy could be saved through effective wear-control measures."

Table 10: Industrial operations with significant annual wear consequences

\begin{tabular}{|c|c|c|}
\hline Industry & Operation & Less masedai), $10^{12} \mathrm{Btu}$ \\
\hline \multirow{5}{*}{$\begin{array}{l}\text { Utilities ( } 28 \% \text { total U.S. } \\
\text { consumption) }\end{array}$} & Seals & 185 \\
\hline & Accessories & 120 \\
\hline & Bearings & 55 \\
\hline & Reliability & 145 \\
\hline & Total & 505 \\
\hline \multirow{7}{*}{$\begin{array}{c}\text { Transportation ( } 26 \% \text { total } \\
\text { U.S. consumption) }\end{array}$} & Brakes & (b) \\
\hline & Valve trains & (b) \\
\hline & Piston ring assemblies & (b) \\
\hline & Trmensmission & (b) \\
\hline & Bearings & (b) \\
\hline & Gears & (b) \\
\hline & Total & (b) \\
\hline \multirow[t]{5}{*}{ Mining } & Ore processing & 22.80 \\
\hline & Surface mining & 13.26 \\
\hline & Shaft mining & 10.70 \\
\hline & Drilling & 5.58 \\
\hline & Total & 52.34 \\
\hline \multirow{3}{*}{ Agriculture } & Tillage & 16.85 \\
\hline & Planting & 2.47 \\
\hline & Total & 19.32 \\
\hline \multirow{3}{*}{ Primary metals } & Hot rolling & 14.30 \\
\hline & Cold rolling & 0.14 \\
\hline & Total & 14.44 \\
\hline
\end{tabular}

Wear is a significant issue in the recovery of renewable energy. Steel components are used throughout infrastructure and equipment. Wind turbine components, specifically steel bearings located in the nacelle, are a great example of the issues of wear in renewable energy. Reports from Sweden, Finland, and Germany between the years of 1997 and 2004 have illustrated both the number of occurrences of specific failures and the downtime to repair the failure. While electrical systems have the highest rate of occurrence, gearbox failures result in the longest overall downtime ( 20 to $30 \%$ of downtime). An average repair to the gearbox takes over 200 hours to repair, and the bearings fail 
more often than any other specific component. Furthermore, a failure to a bearing can often result in the repair or replacement with other components adjacent to the bearing. From the results reported in Sweden, the average turbine has a failure 0.402 times a year. In 2012, the United States brought the total wind energy capacity to $60 \mathrm{GW}$. $60 \mathrm{GW}$ represents $\$ 120 \mathrm{~B}$ of investment, the equivalent power required for 14.7 million homes, avoids the consumption of 36.6 billion gallons of water, and avoids 95.9 million tons of $\mathrm{CO}_{2}$. If we assume the U.S. has similar failures to Europe, and then look at the total amount of down time due to only gearbox failures, we would be looking at $\sim 0.08$ times a year.

For the $60 \mathrm{GW}$ of installed power in 2012 that equates to $0.55 \mathrm{GW}$ of lost generation capacity due to wear. The growth curve for installed wind power generation is shown in Figure 29 and is expected to continue. Clearly enhanced bearings will have a significant impact on energy savings.

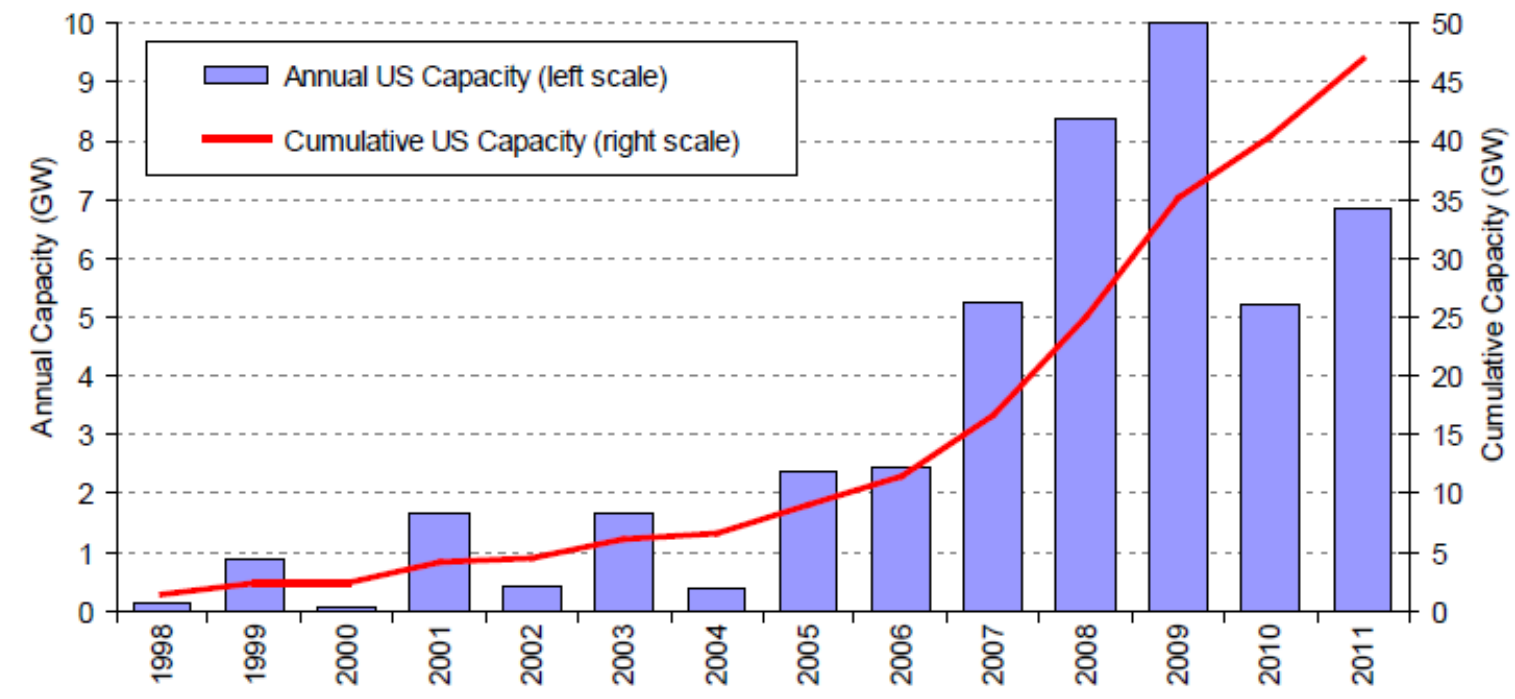

Source: AWEA project database

Figure 29. US wind power market growth curve from 1998 to 2011. 


\section{COMMERCIALIZATION \& RECOMMENDATIONS}

The fabrication of steel components from powder metallurgy is a mature technology that is used in high performance applications such as tooling, biomedical components, automotive, aircraft, appliances, and energy production figure 30). Powder production via gas atomization and consolidation of powders by canning and hot isostatic pressing has been commercialized by companies such as Carpenter Technology Corporation, one of this project's main industrial collaborators. The estimated sales of PM components for North America alone are over \$7 billion, and represent 25,000 jobs (figure 31). The objective of this project was to determine and provide information on the potential benefits from PM microstructures over casting microstructures that may improve PM bearings and other components for application in wind energy generation and other energy industries. In the Metal Powder Industries Federation's "PM Industry Roadmap" written in 2012, alternative energy is listed as one market that represents a significant opportunity for PM industry. Wind energy is the first renewable discussed in this report, and bearings and gears are listed as components required by this industry that could be fabricated using PM.

\section{PM Structural Components Markets}

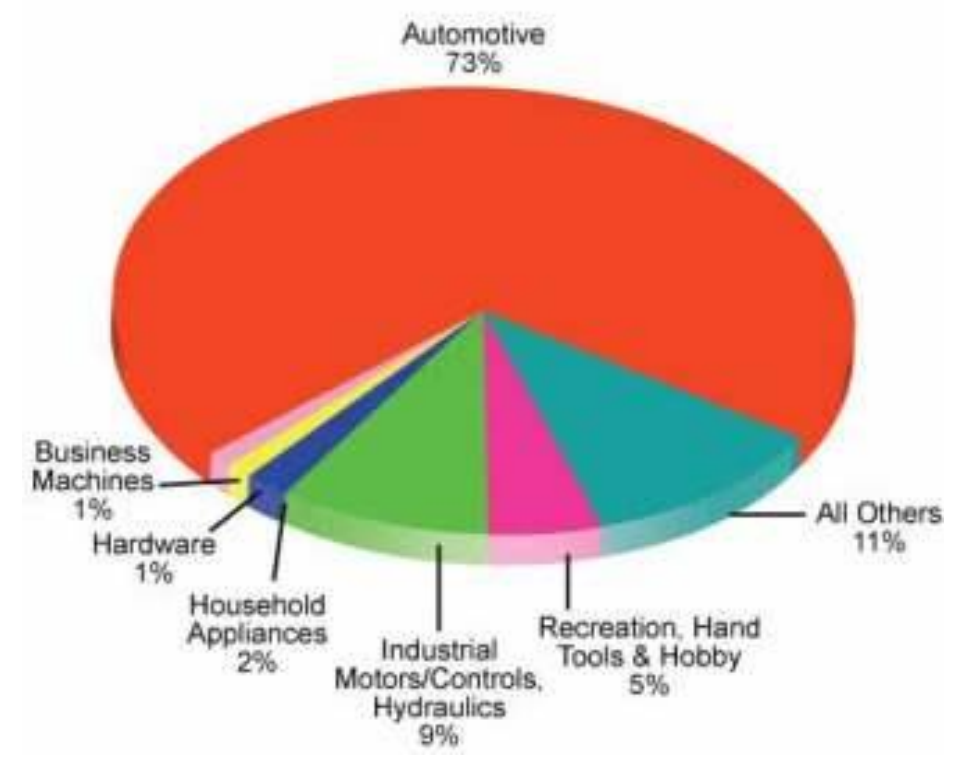

Figure 30. The powder metallurgy structural components markets. 


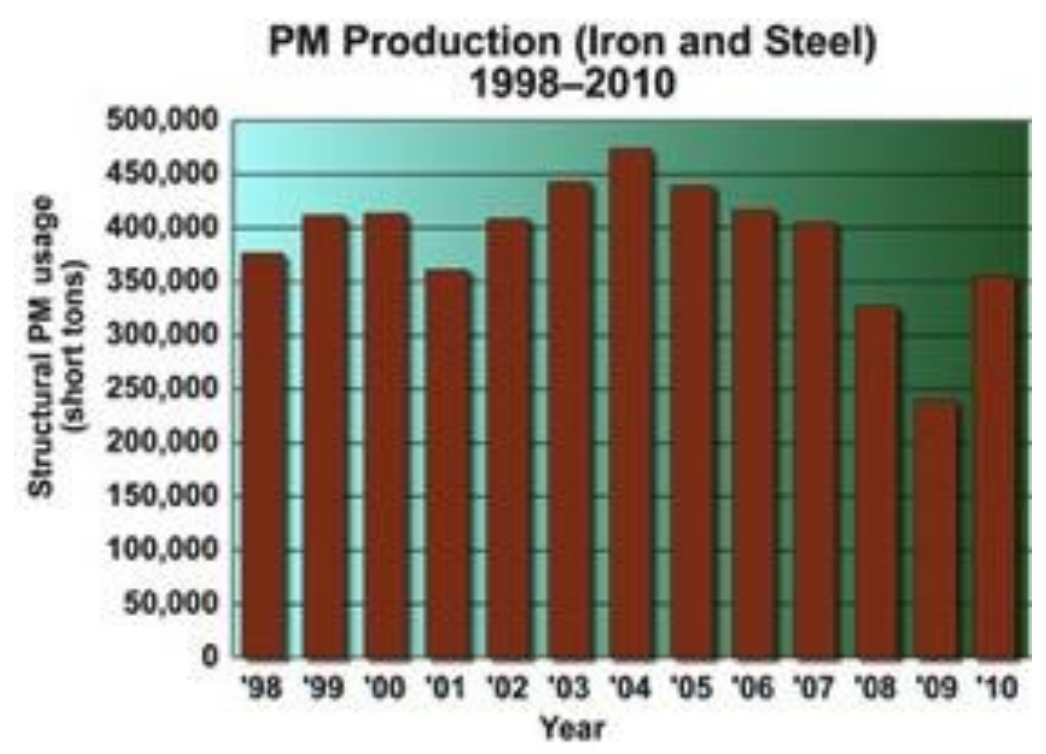

Figure 31. Production of iron and steel from powder metallurgy 1998-2010.

The early results found in this project illustrate some advantages of component enhancements from the PM microstructures compared to the cast microstructures for the given fabrication procedures and testing conditions as will be further discussed in conclusions. However, several key evaluations should be performed beyond the current scope of this project that may better illustrate the advantages for PM in this area of application. Some of the alloys exhibited improved micro-pitting wear resistance over cast alloys; this area needs to be further explored to better understand how and if this will translate into increased longevity of PM bearings. In addition, a larger range of commercial alloys being evaluated may better characterize the difference in properties. Powder metallurgy allows fabrication of graded structures. For example, a bearing could be fabricated with ceramic particles in a metal matrix composite at the surface of the bearing in any wear prone areas. For the scope of this project the team tried to stay with alloys that were similar between castings and powder metallurgy. Further testing should be performed with alloys only possible through solid state processing. Examples of potential alloys that could be evaluated include the nanocomposite materials recently highlighted in Scientific American [12]. Metal matrix composites and alloys with high concentrations of interstitials are possible with powder metallurgy and could further improve the wear resistance.

In this project, only the bearing materials were investigated; changing lubrications and the differential hardness between rollers and discs (bearings and gears) would also provide further understanding on the performance of powder metallurgy materials. The test samples used in this project were from samples that were from small castings or PM samples. Many bearings would be made from much larger castings. The microstructures would vary from the outside surface to the center of the casting due to the large thermal gradients and inconsistent cooling rates; these differences were not evaluated in this testing. Finally, the test methods investigated in this project were chosen due to the comparative ease of testing, ability to evaluate multiple modes of wear, and elimination of risk to wind turbine equipment. However, field testing may provide further evidence as to the difference in materials. A laboratory test cannot fully capture field conditions, and events, contaminates, debris, or aggressive environments could modify the results witnessed in a wind turbine. After further laboratory evaluation, field tests would provide valuable feedback as to the accuracy of the laboratory testing. 


\section{ACCOMPLISHMENTS}

Following accomplishments have been made possible through the present project;

- Installed a computer-controlled micro-pitting test rig (MPR) in the tribology laboratory of ORNL's Materials Science and Technology Division, and developed wear mechanism to see high cycle rolling contact fatigue characteristics under lubricated environments.

- Developed test geometry and machining practices with MPR to perform work on these wear resistant alloys which simulate the actual wear behavior (i.e. micro-pitting) of the bearing materials in a wind turbine gear box.

- Tested total 5 different alloys/ 15 specimens to determine the benefit of power metallurgy on the wear resistant alloys to be used as bearing materials.

- One extended abstract with the following title was published.

P. J. Blau, Y. Yamamoto, K. M. Cooley, and S. Reeves, "INVESTIGATION and CHARACTERIZATION of MICRO-PITTING DAMAGE in BEARING STEELS"

An oral presentation with the same title above was presented for a meeting "Society of Tribologists and Lubrication Engineers (STLE) Annual Meeting 2013," which was held at Detroit, MI in May 2013. 


\section{CONCLUSION}

Oak Ridge National Laboratory, in Partnership with Avure Technologies Inc. and Carpenter Technology, has investigated the potential for improving the wear resistance of wind turbine gearbox bearings by fabrication using the Hot Isostatic Pressing (HIP) of high alloy steel powders. Three candidate bearing steels $440 \mathrm{C}, 440 \mathrm{XH}$, and M62, were selected for this processing study. A commercial high chromium bearing steel, AISI 52100, and a common tool steel, M50, were also included for comparison. All five steels, the three test steels and the two reference steels, were hardened by established heat-treatment practices. Wind turbine gearbox makers have recently focused on reducing micro-pitting, an early form of surface damage that can lead to more severe problems. Therefore, a durability test method specifically designed to evaluate micro-pitting behavior under lubricated rolling contact conditions was employed in this work. The resultant wear test data, coupled with surface observations, were used to compare the micro-pitting characteristics of microstructurally well-characterized high alloy steels. Information developed in this work was intended to determine the extent to which the application of advanced HIP process can improve bearing durability in contact fatigue environments like wind turbine gearboxes. Of the five steels tested, the 440XH was particularly resistant to micro-pitting in comparison to the 52100 steel which exhibited extensive surface damage. Under lubricated conditions, the relative roles of the materials, the surface roughness, and the lubricant properties of friction and wear must be taken into consideration. The current work has focused the role of the materials by using a relatively poor lubricant.

First trial rolling-sliding testing with relatively larger slip ratio (20\%) and lower load (305N) resulted in showing wear behavior as a dominant mode, rather than micro-pitting feature. The M50 showed the largest displacement (or the amount of surface wear) among the tested materials, but this test condition could not differentiate the wear characteristic or resistance of the other materials. Crosssectional observation of tested surface indicated the micro-crack initiation associated with existing carbides.

Optimized rolling-sliding experiments with relatively smaller slip ratio (5\%) and higher load (650N) exhibited signs of abrasive wear, but not all had the same degree of micro-pitting damage. The 52100 steel had the most severe micro-pitting, consisting of distorted craters, many of which were connected by micro-cracks. Cross-sectional examination showed multiple micro-cracks extending into the alloy microstructure from the same pit, and the depth of the cracks was several times the depth of the micro-pit. Type 440XH had the least evident micro-pitting damage. Pits were more localized and the micro-cracks associated with them tended to avoid carbides during propagation. Abrasive processes were produced by debris from mico-pitting, and the two processes could work together to worsen the surface damage. A system of progressive sliding and rolling contact damage was proposed. There was no evidence for the formation of what are called 'white layers' in the current micro-pitted specimens, in contrast to such layers that are found in conjunction with macro-pitting of rolling element bearings and gears.

All these results suggested the potential advantage of high alloy tool steels, such as $440 \mathrm{XH}$, for a better resistance of micro-pitting features, compared to conventional cast-and-wrought type tool steels with relatively low alloying elements. Only PM process can realize the optimization of microstructure of the high alloy tool steels suitable for the expected properties 


\section{REFERENCES}

[1] D. W. Hetznera, W.V. Geertruydenb, "Crystallography and metallography of carbides in high alloy steels", Materials Characterization, vol. 59 (2008) pp. 825-841.

[2] ASTM G-40-12 Standard Terminology Relating to Wear and Erosion, ASTM Annual Book of Standards, Vol. 03.02

[3] P. J. Blau (1989) Friction and Wear Transitions of Materials: Break-in, Wear-in, Run-in, Noyes Pub., Park Ridge, NJ.

[4] P. J. Blau (2010) "Wear Analysis of Wind Turbine Gearbox Bearings," Oak Ridge National Laboratory Technical Report ORNL/TM-2010/59, (figure 1001SK02), p 22.

[5] T. S. Eyre and D. Maynard (1971) "Surface aspects of metal-to-metal wear," Wear, Vol. 18(4), pp. 301-310.

[6] T. S. Eyre and A. Baxter (1972) "The formation of white layers at rubbing surfaces," Tribology, Vol. 5 (6), pp. 256-261.

[7] P. J. Blau (1985) "Measurements and Interpretations of Sliding Wear Damage in Metals," J. of Tribology, Vol. 107, p. 483.

[8] P. J. Blau and A. D. Doyle (1987) "Metallographic Evidence for the Nucleation of Subsurface Microcracks During Unlubricated Sliding of Metals," Wear, Vol. 117, p. 381.

[9] Y. Y. Yang, H. S. Fang, and W. G. Huang (1996) "A study on wear resistance of the white layer," Trib. International, Vol. 29 (5), pp. 425-428.

[10] G. Baumann, H. J. Fecht, and S. Liebelt (1996) "Formation of white-etching layers on rail treads," Wear, Vol. 191 (1-2), pp. 133-140.

[11] Surface Engineering for Corrosion and Wear Resistance. J.R. Davis Ed. ASM International, Materials Park, OH. 2001.

[12] 9 Materials That Will Change the Future of Manufacturing, Scientific American, Steven Ashley and Larry Greenemeier, 2013, http://www.scientificamerican.com/slideshow.cfm?id=9materials-that-will-change-manufacturing 